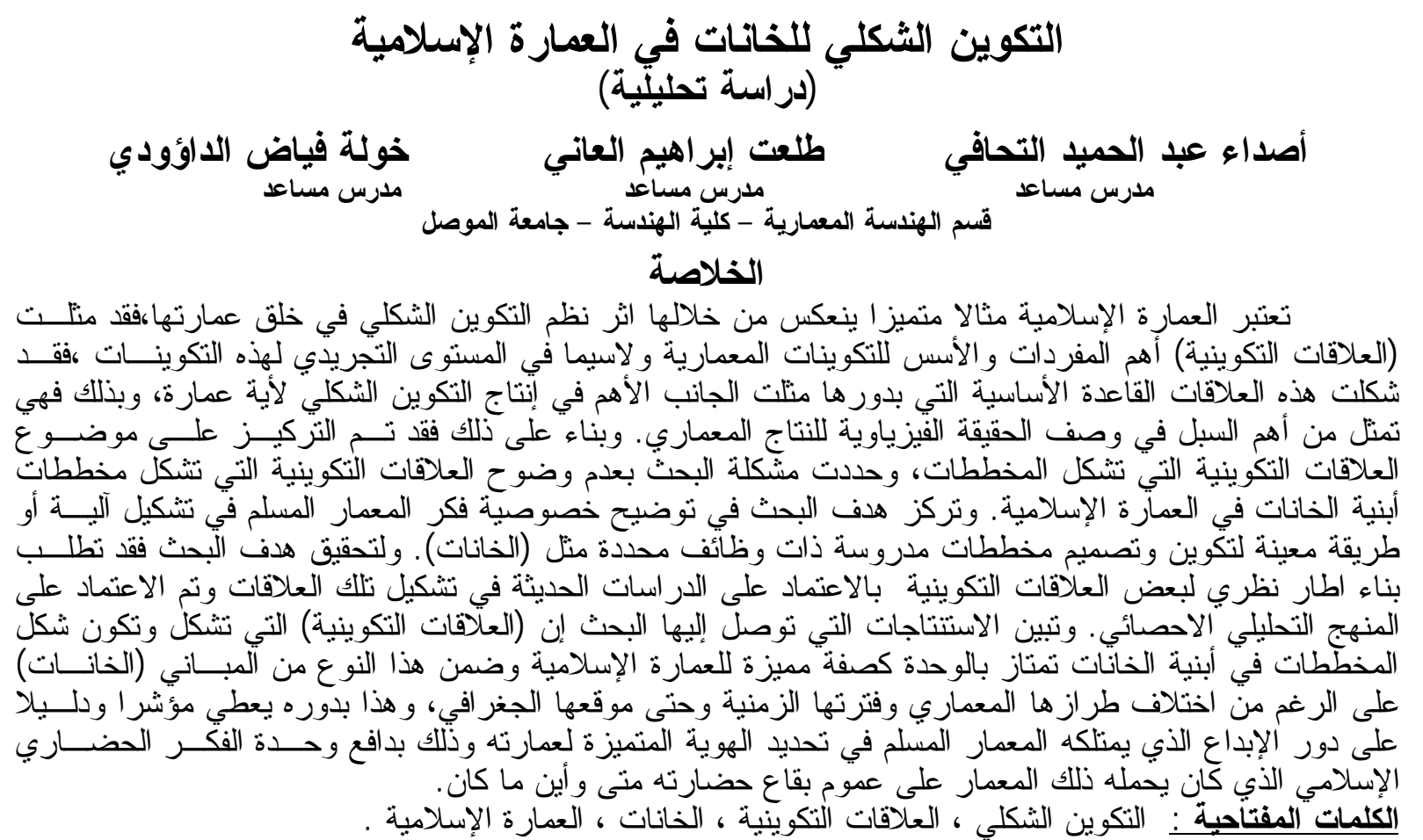

\title{
The Formal Composition of Khans in Islamic Architecture (An Analytical Study)
}

\author{
Khawla F. M. Al-Daudi Talaat I. M. Al-Aane Assda A. H. Al-Tuhafi \\ Assistant lecturer \\ Assistant lecturer \\ Assistant lecturer \\ Engineering Col. - Architecture Dept. - University of Musol
}

\begin{abstract}
Islamic architecture is considered to be a unique example that reflects the impact of structural formation systems in establishing its form; the structural relations represent the most important items and bases of architectural structures, especially at the abstract level. The structural relations form the basic foundation which represent the most important aspect in producing the formal structure of any building thus they are considered to be the most important method in describing the physical reality of architectural production . Stemming from the above fact, the study focuses on the structural relations that form the plans. The problem of the study is that structural relations which form the plans of Khans buildings in Islamic architecture are not clear. The aim of the present study is to clarify the specialty of Muslim architect intellectuality in the formation of a particular mechanism or method to form and design studied plans with specific functions like khans. To achieve the aim of the study a theoretical framework of structural relations is built depending on modern studies on formation of those relations on the bases of the statistical analytical method. The results arrived at show that the structural relations which form and construct the plans of Khans buildings are characterized by uniqueness as a distinguishing character of Islamic architecture some of these types of building are the khans, though they differ in their architectural design, their date and ever their geographical location. This in turn gives an indication and proof of the Muslim architect creative role in determine the distinguished identity of his architecture with the incentive of Islamic cultural intellect held by that architect all over the places of his civilization whenever and wherever he exists.
\end{abstract}

Key Word : Formal Composition, Relationships Formative, Khan, Islamic architecture . 


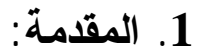

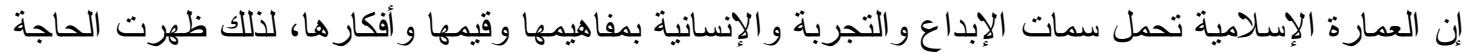

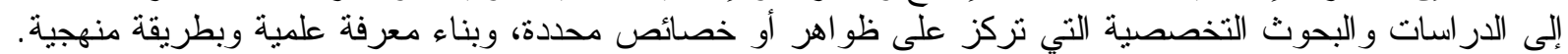

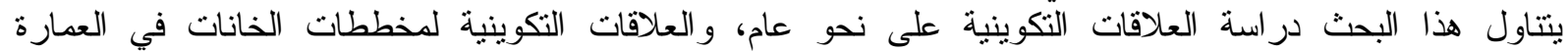

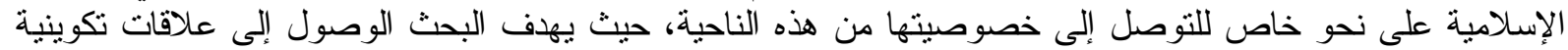
لمخططات الخانات في العمارة الإسلامية وذلك عبر منهجية تقوم على الدراسة التحليلية و الإحصائية، هذه العلاقات تلبين الإلين

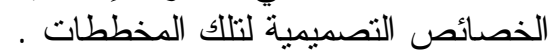

1.1 التكوين الثكلي للمخططات في العمارة:

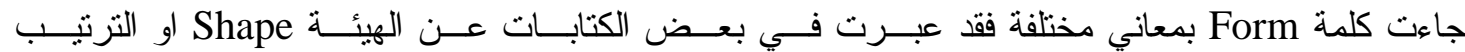
Configuration الم System of Relations

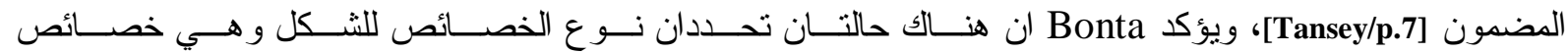

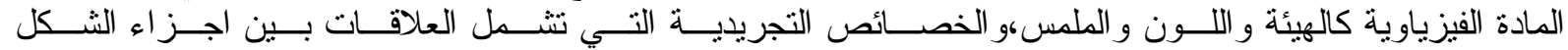

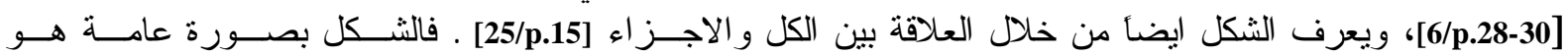

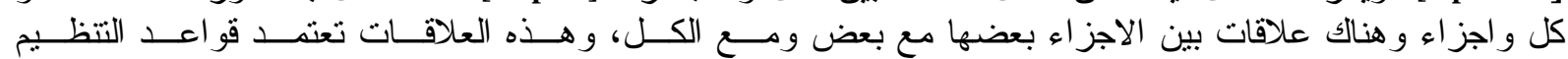

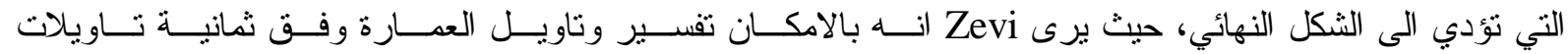

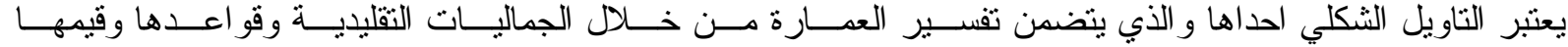

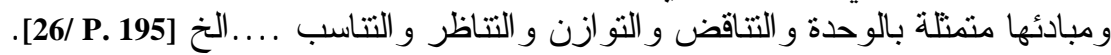
تعد العلاقات التكوينية من المفاهيم المهمة في النظرية المعمارية، ومحور التئية رئيسيا من المحاور التي نت تناولها

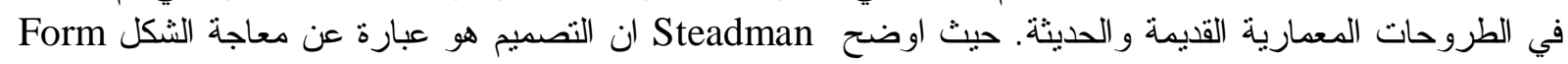

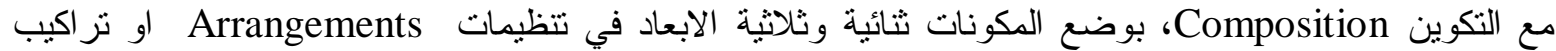
20/p.2] Configurations

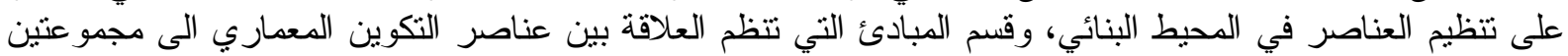

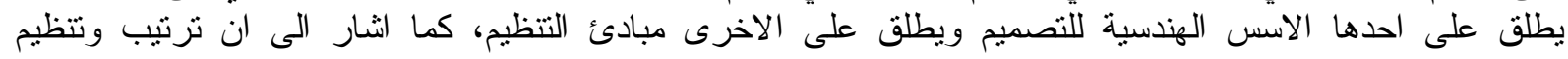

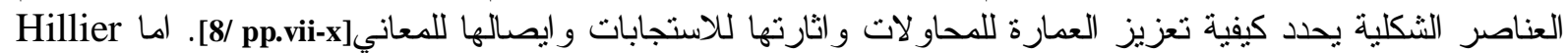

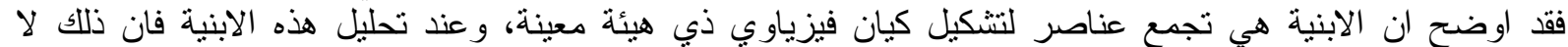

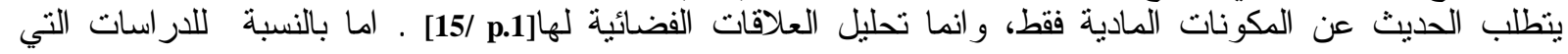

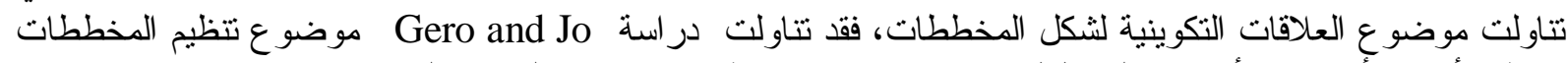

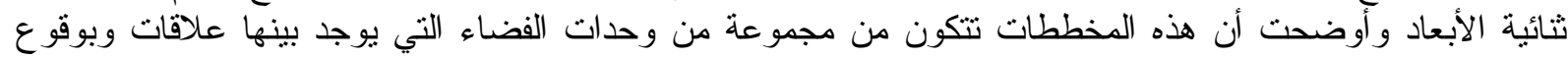

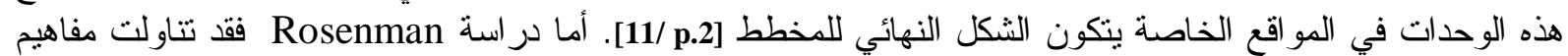
طريقة توليد حلول تصميمية داخل نظام منكر ج لمخططات ثنائية الأبعاد من خلال تركيب وحدة فضاء التهاء أساسية تكون

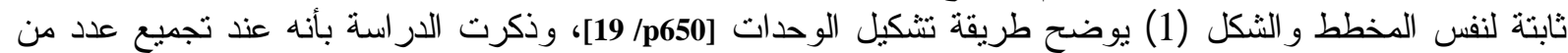

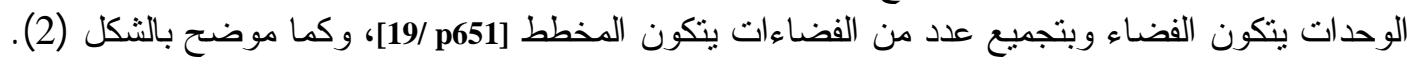
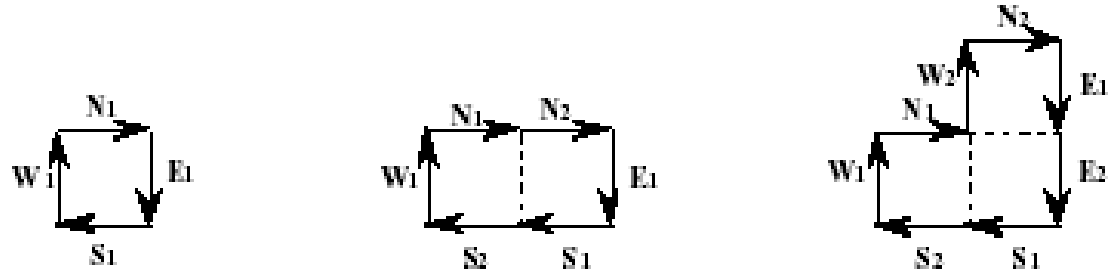

الثكل (1): طريقة تشكيل الوحدات [19/p650]. 


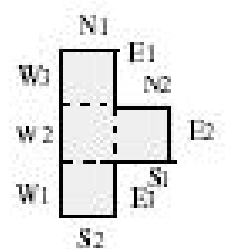

B الوحدة

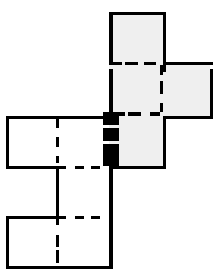

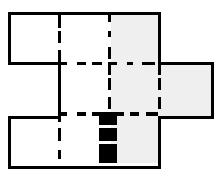

علاقة التنلاصق

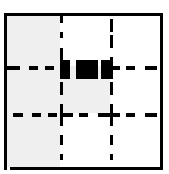

علاقة التراكب

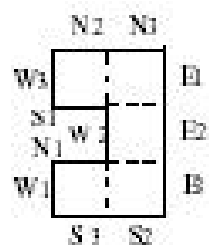

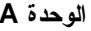
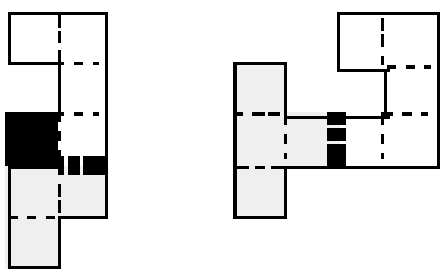

الثكل (2) : العلاقات التكوينية الممكنة بين الوحدات [19651/p2]ـ.

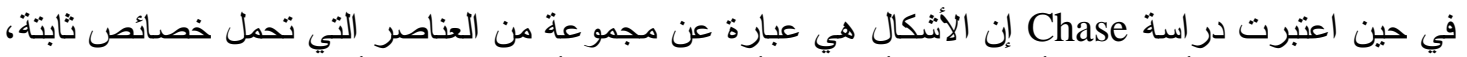

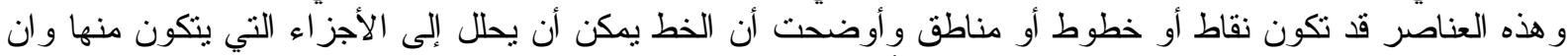

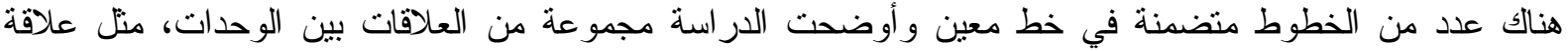

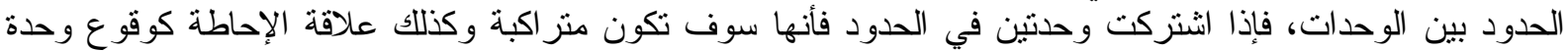

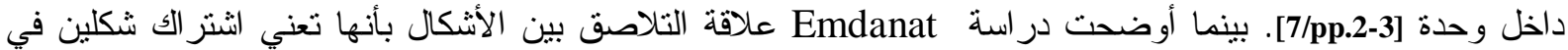

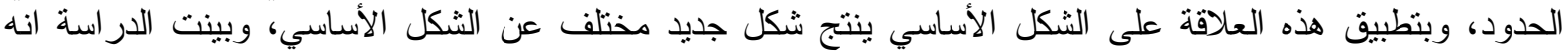

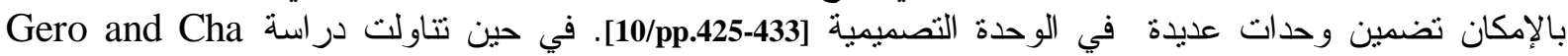

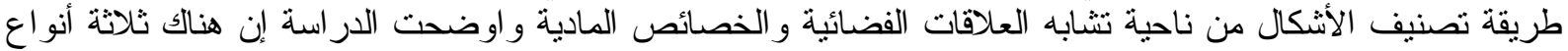

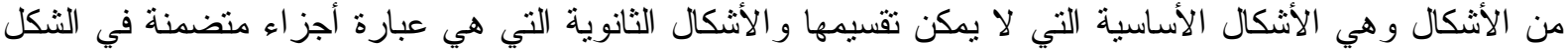

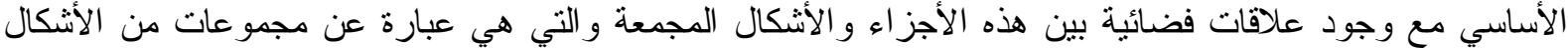

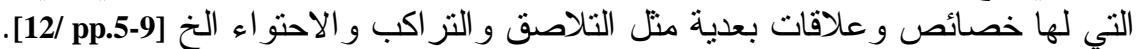
من خلال مناقثة الدراسات السابقة برزت عدة ولتهات مفردات ارتبطت بطبيعة العلاقات التكوينية للمخططات،

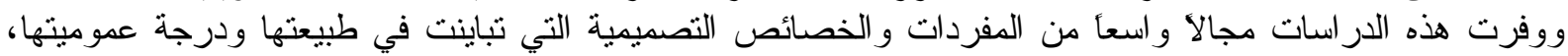

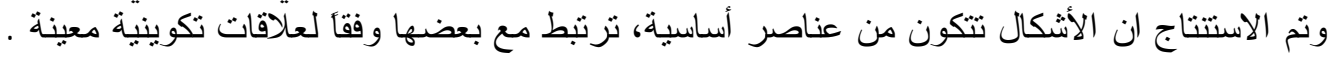

2.1 التكوين الثكلي للمخططات في العمارة الاسلامية:

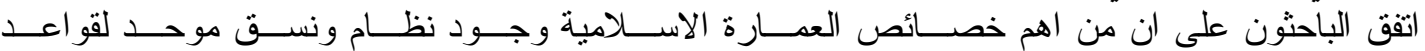

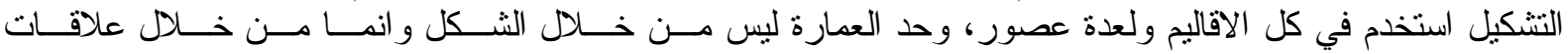

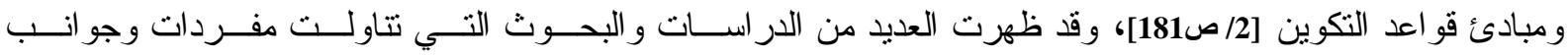

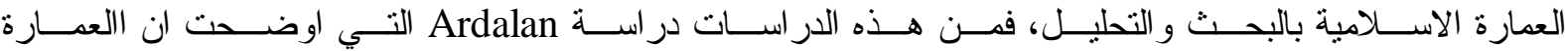

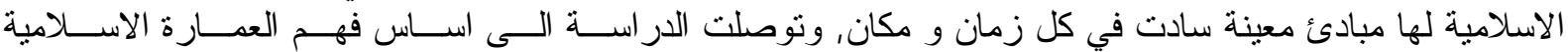

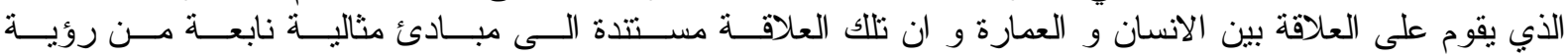

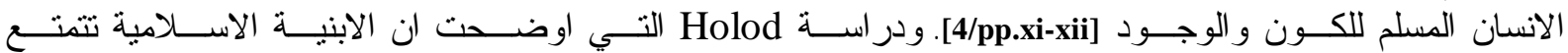

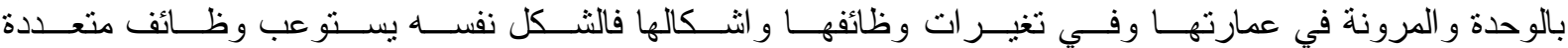

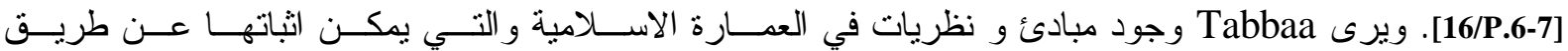

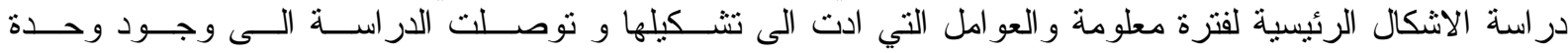

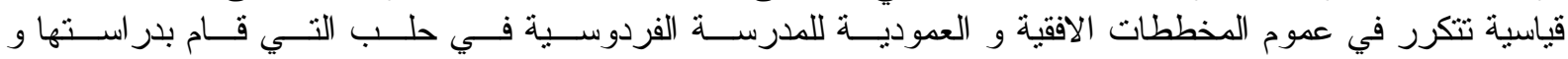

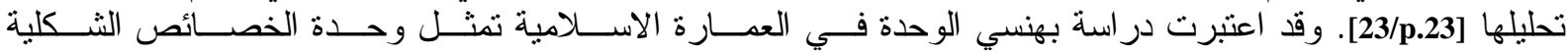

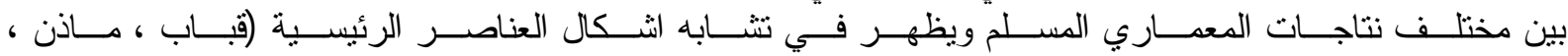




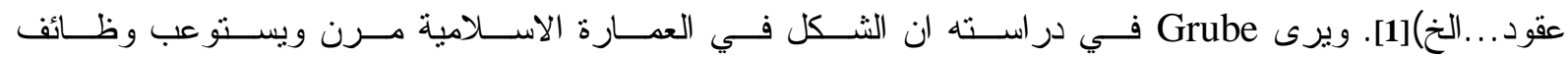

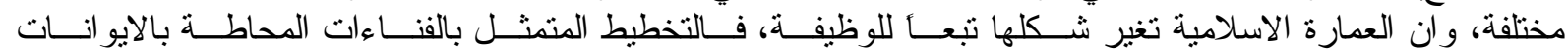

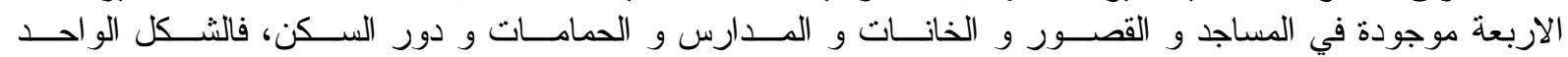

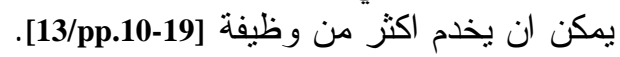

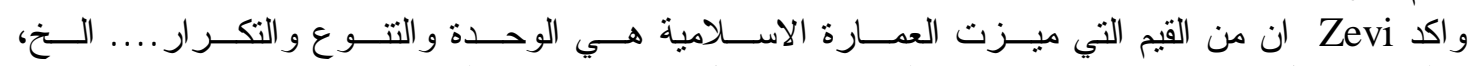

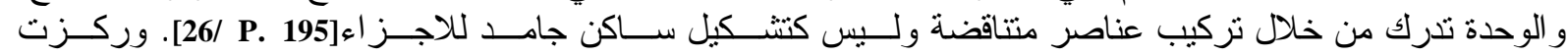

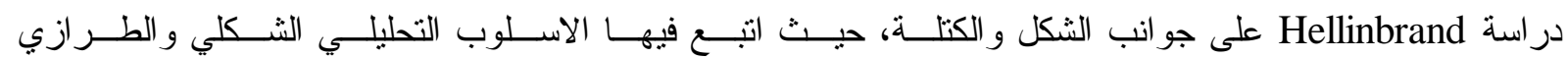
للانماط الوظيفية في العمارة الاسلامية [14/p.vi

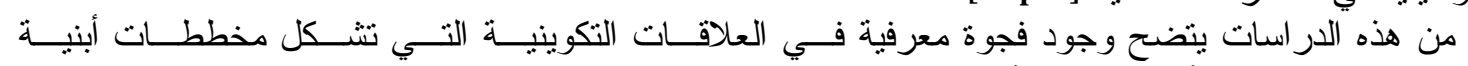

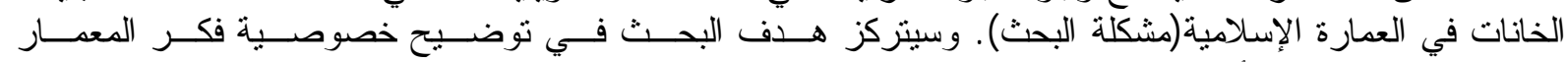

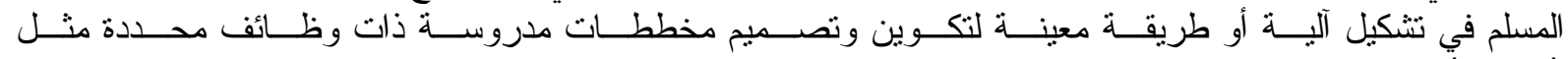

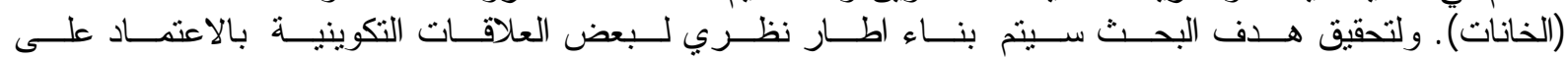

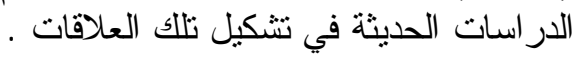

3.1 الخانات في العمارة الإسلامية:

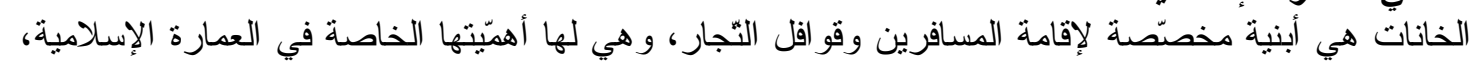

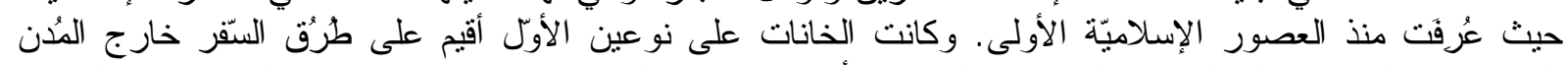

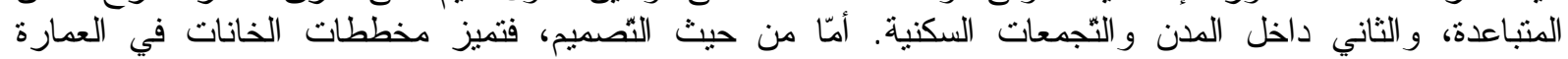

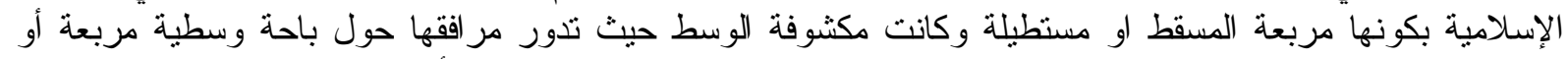

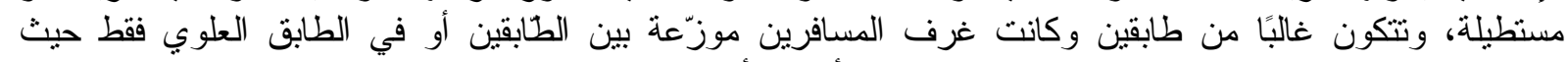

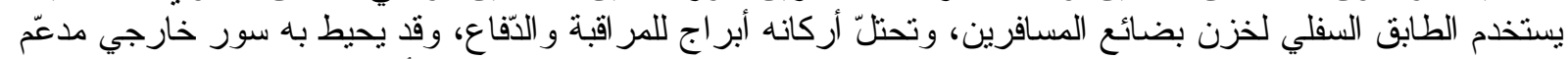

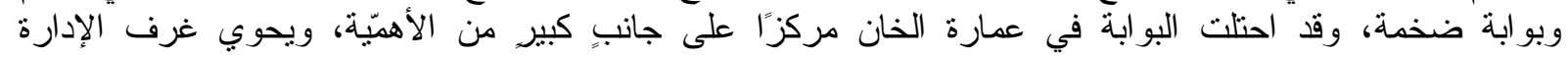

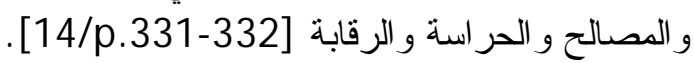

2. 2 2.2 تحديا المتغير ات: 1.2 بعض المفاهيم المستخدمة في تكوين العضاءة العلاقات الشكلية للمخطط:

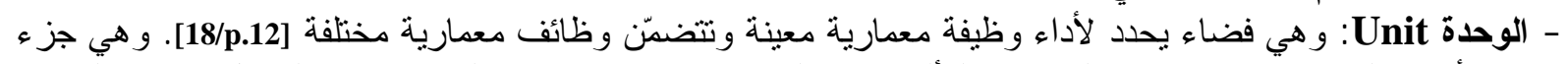

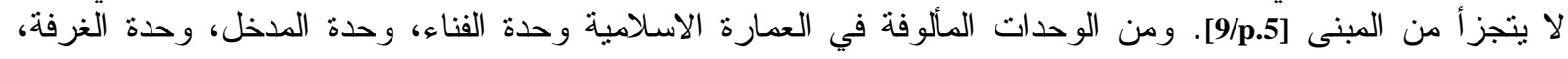

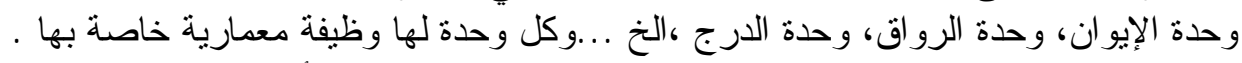

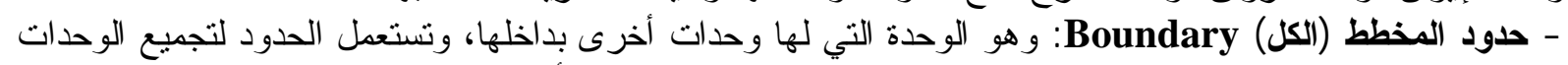

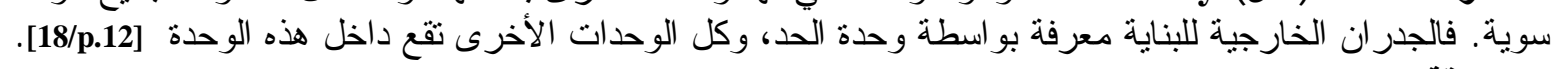

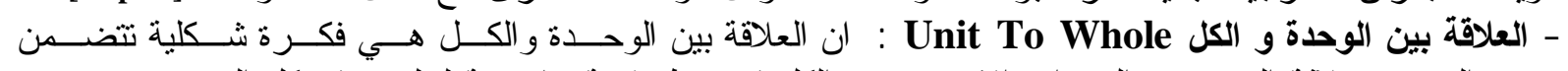

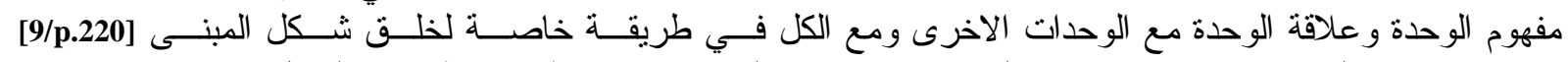

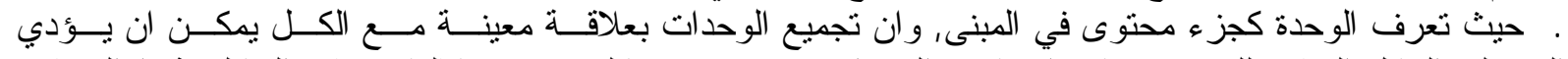

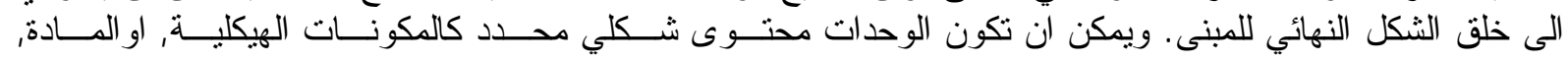

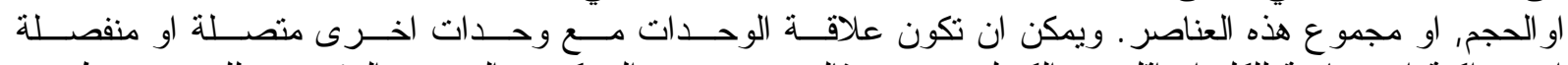

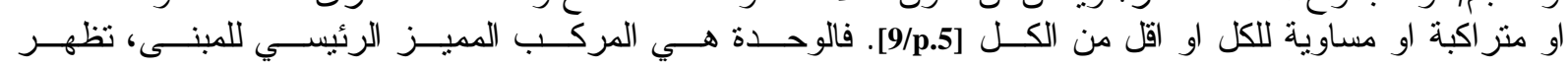

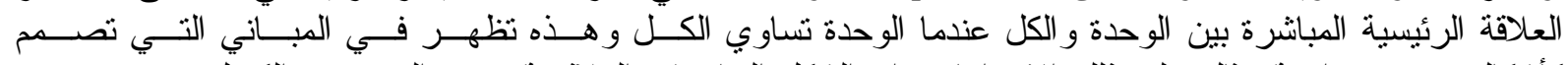

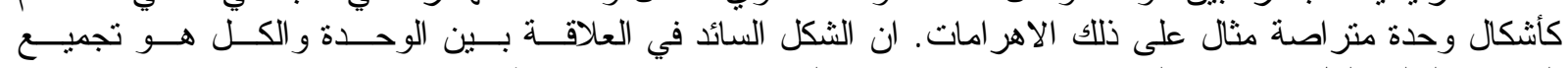

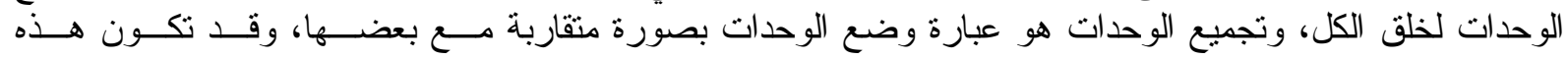

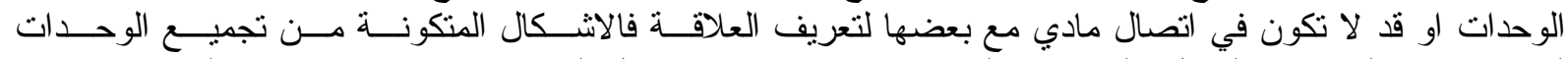

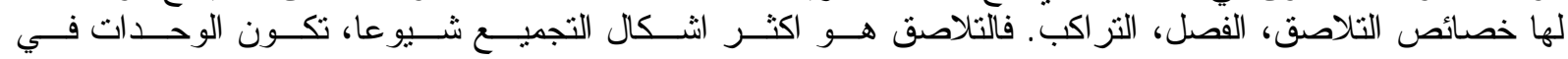

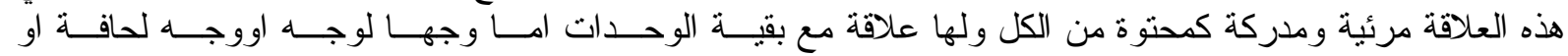

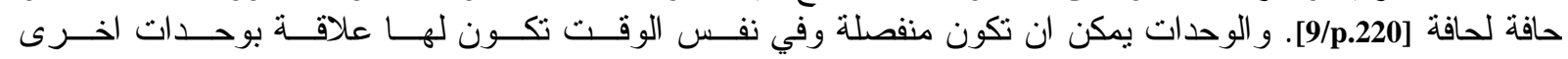

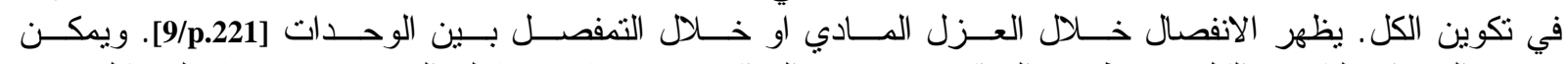

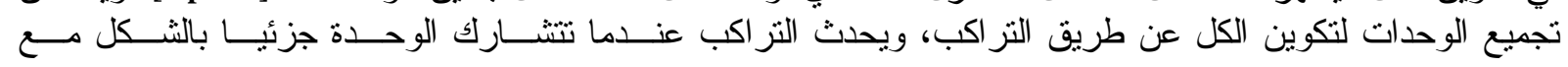




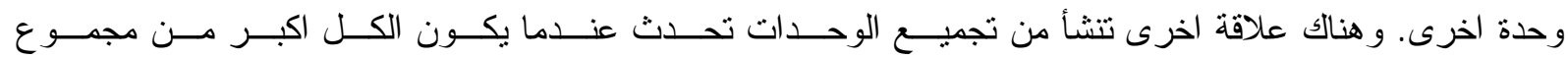

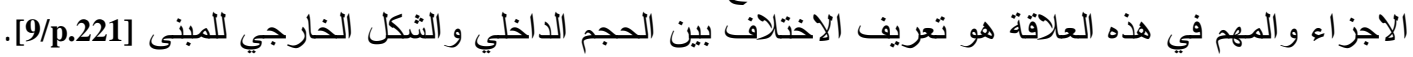

2.2 العلاقات التكوينية المنتخبة لتنظيم المخططات: ترتبط العلاقات التكوينية بخصائص المخطط و الوحدات التي تشكل المخطط بدون الاعتماد على الأبكاد

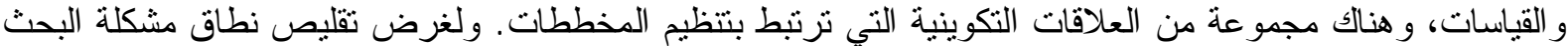

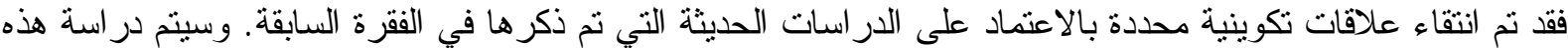

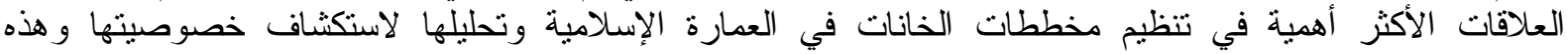

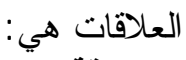

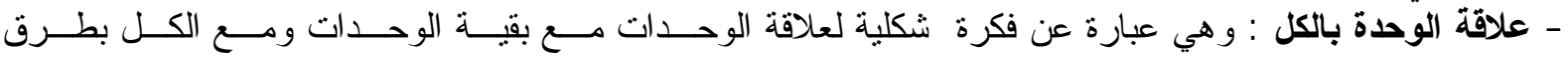

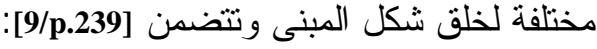
- مفهوم الوحدة بحد ذاتها.

- مفهوم وحدات منر ابطة مع غيرة فير ها لخلق شكل أو بنية معينة.

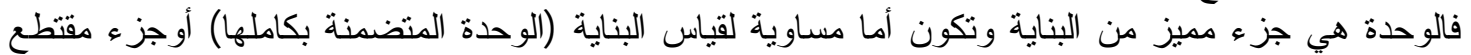

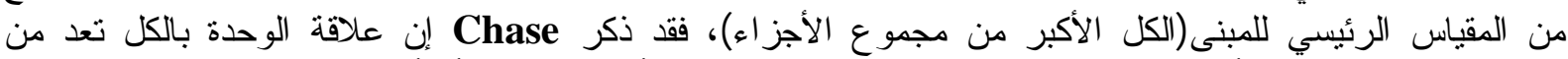

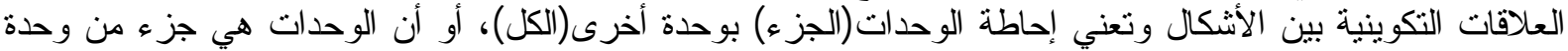

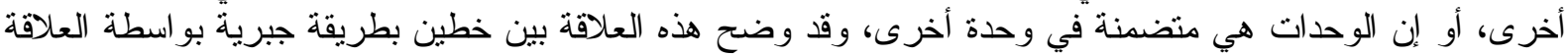

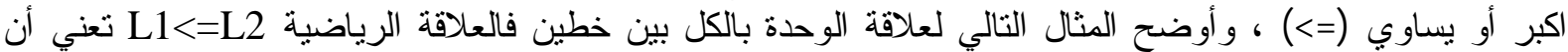
الخط L1 هو جزء من الخط L2 [7/p.3]. كما ذكر Medjdoub أن علاقة الوحدة بالكل تكون بين وحدنين احدهما

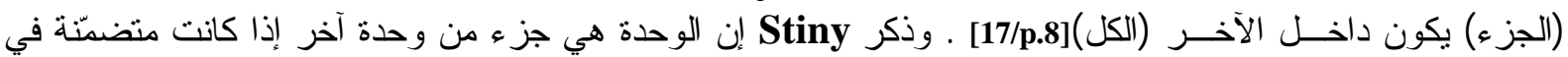

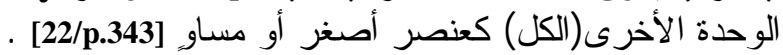
وسيتم انتخاب مجموعة من العلاقات التكوينية بين الوحدة و الكل والوات وفقا للمستويات الآتية: 1.2.2 مستوى الكل: ويشمل المفردات التالية:

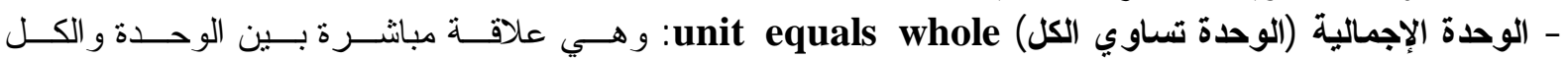

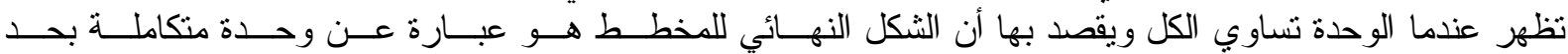

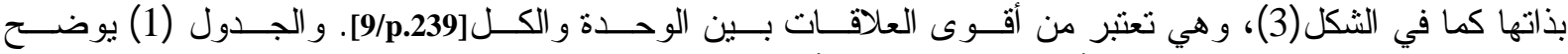

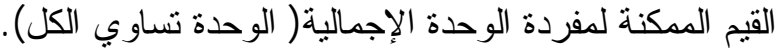

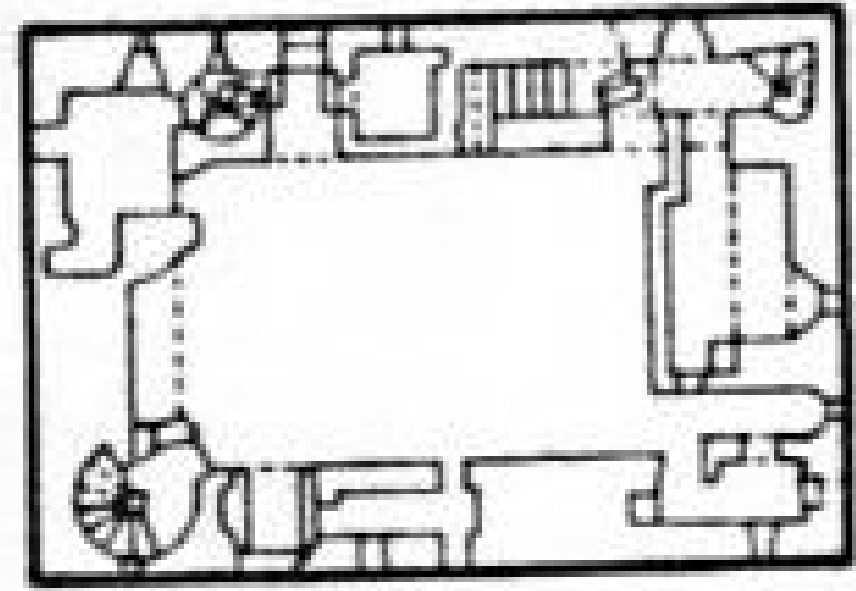

الثكل(3) مبنى Elphinstone Tower يمثل الوحدة الإجمالية [9/p.239]. 


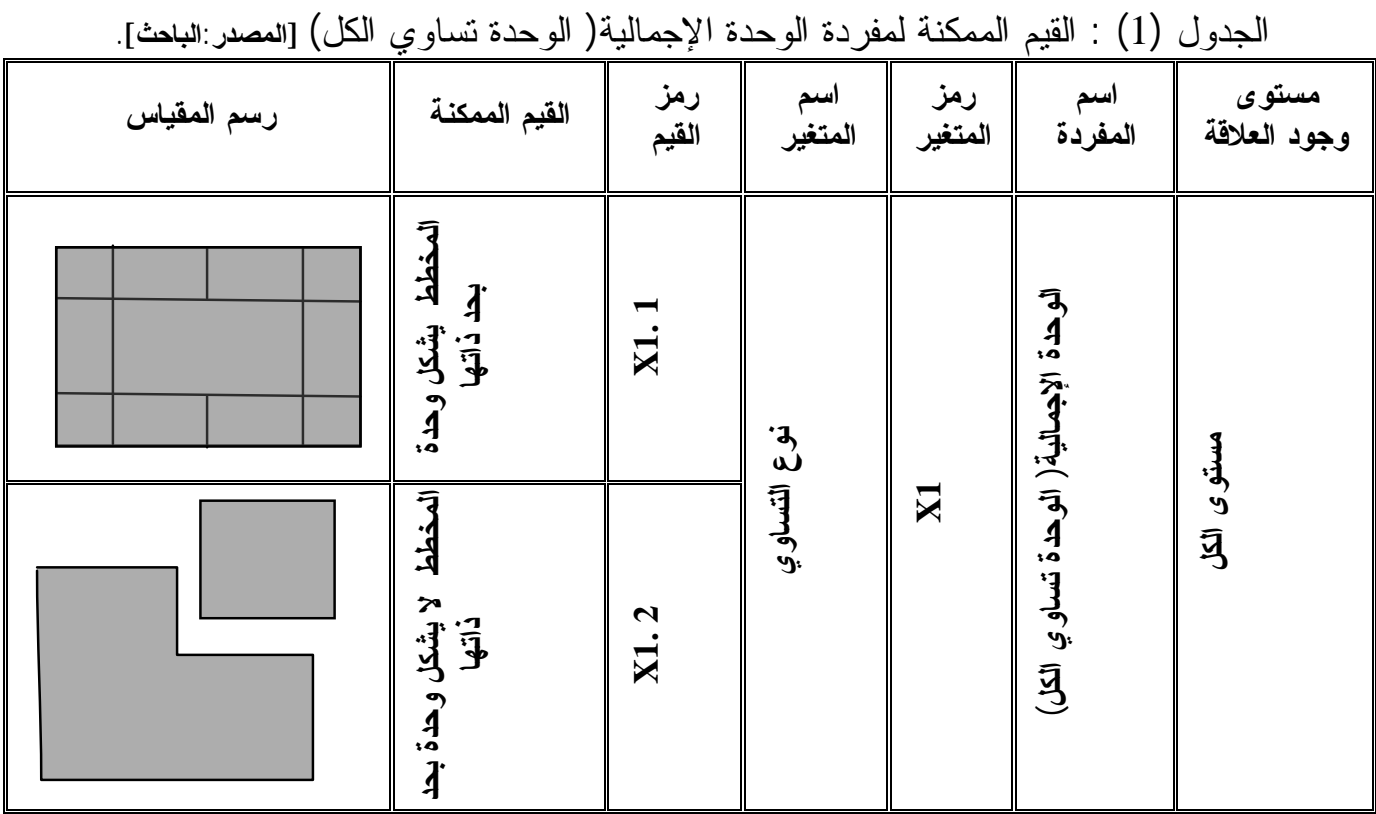

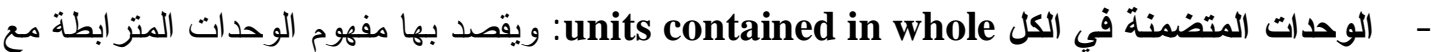

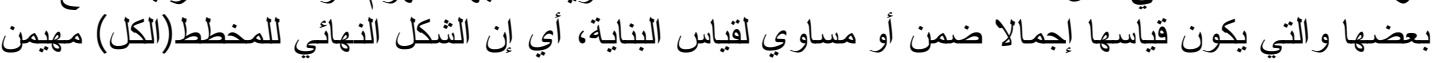

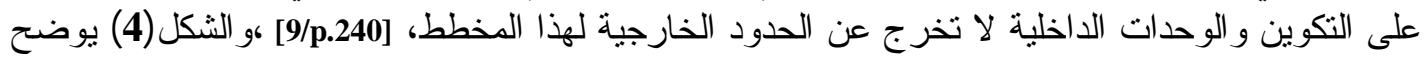
ذلك، أما الجدول (2) فيوضح القيم الممكنة لمفردة الوحدة المتضنة الأنة في الكل.

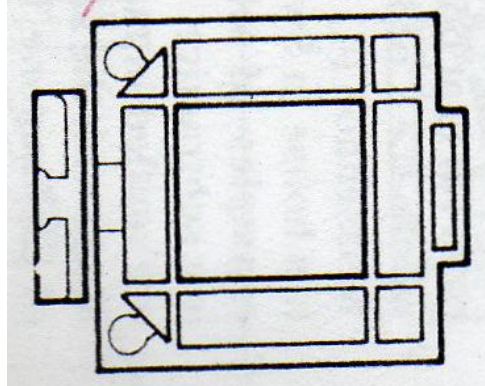

الشكل (4) مبنى19/p.240].st.Mary Woolnoth

2.2.2 مستوى علاقة الجزء مع الكل: ونتثمل المفردات التالية:

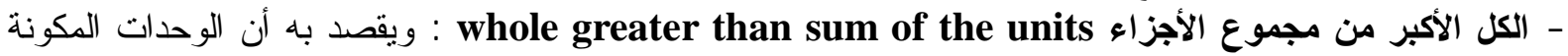

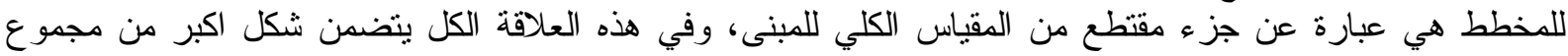
الأجزاء المكونة له وكما موضح بالثكل(5) [9/p.241]. أما الجدول (3) فيوضح القيم المكنة لمفردة الكل الأكبر من

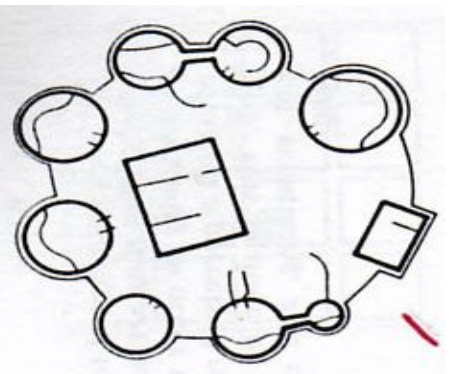

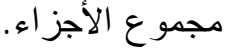

الشكل(5) مبنى 9/p.241] Musgum Village.

الجدول (2) : القيم الممكنة لمفردة الوحدة المتضمنة في الكل [المصدر:الباحث]. 
العاني : التكوين الشكلي للخانات في العمارة الإسلامية (در اسة تحليلية)

\begin{tabular}{|c|c|c|c|c|c|c|}
\hline رسم المقياس & القيم الممكنة & رلقيم & المتغير & المتغير & المفردة & مستوى وجود \\
\hline & 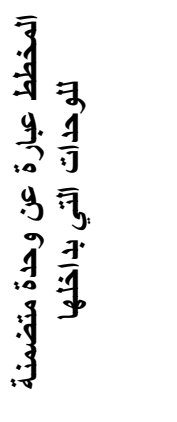 & $\tilde{\tilde{x}}$ & \multirow[t]{2}{*}{$\begin{array}{l}3 \\
\text { 年 } \\
\text { 司 } \\
3 \\
3\end{array}$} & \multirow[t]{2}{*}{$\tilde{x}$} & \multirow{2}{*}{ 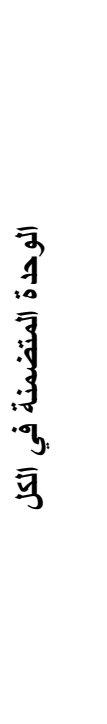 } & \multirow[t]{2}{*}{$\begin{array}{l}3 \\
\text { 3. } \\
\text { y } \\
\text { ⿹ }\end{array}$} \\
\hline & 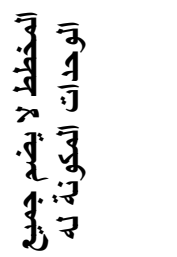 & $\tilde{\check{X}}$ & & & & \\
\hline
\end{tabular}

الجدول (3) : القيم الممكنة لمفردة الكل الأكبر من مجموع الأجز اء [المصدر:الباحث].

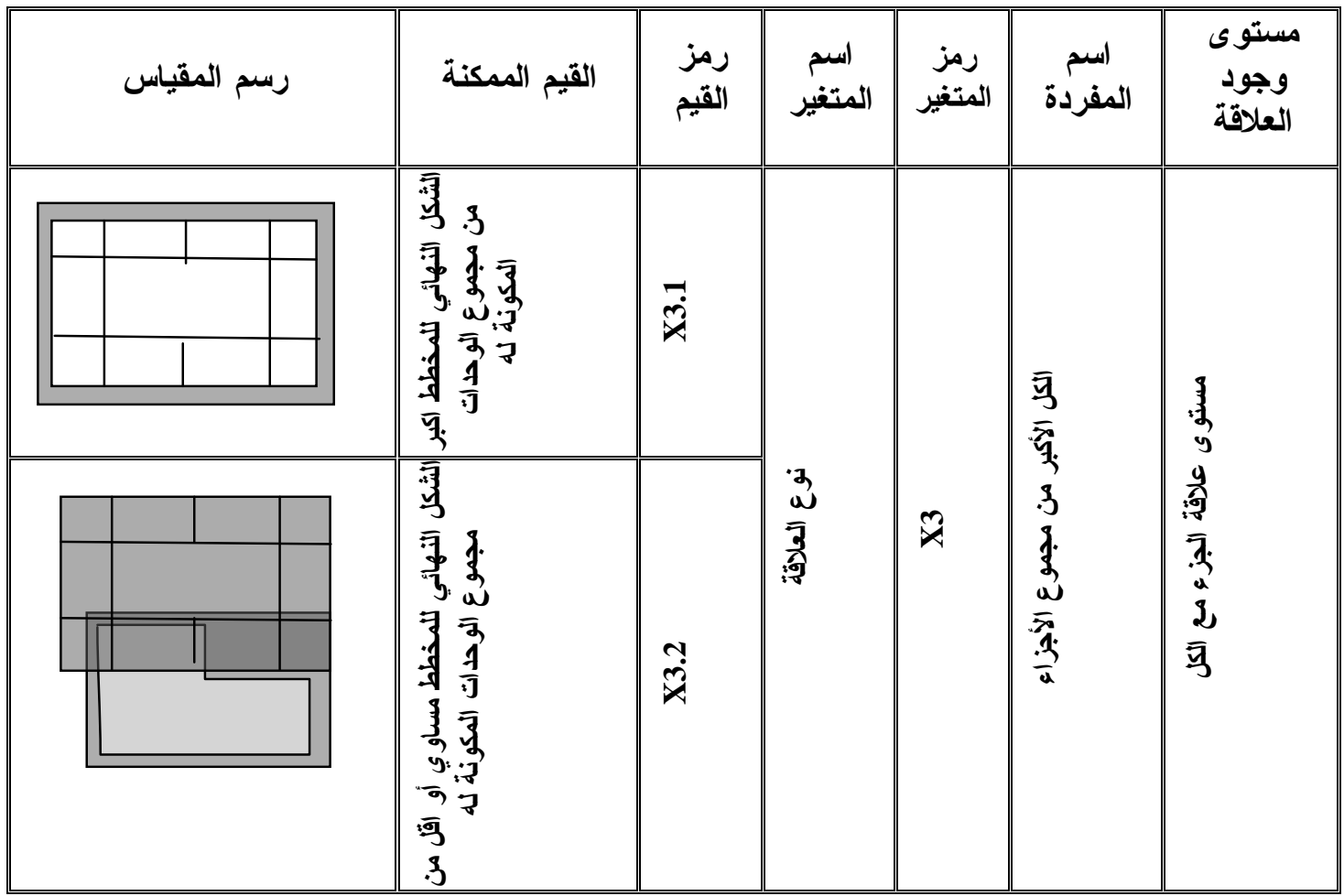

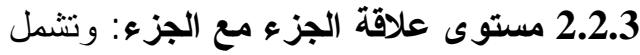

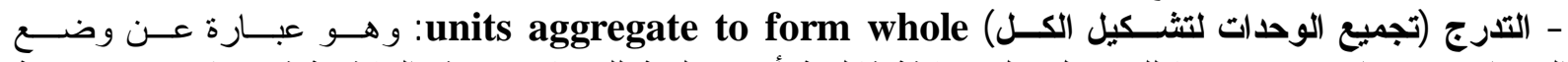

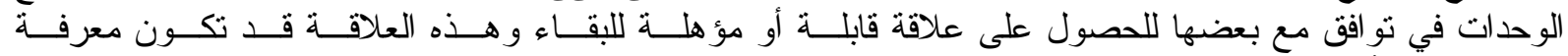

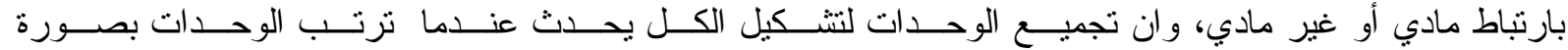

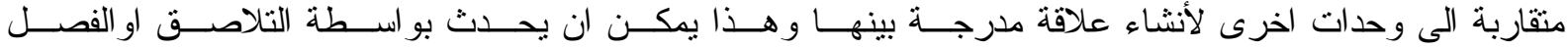

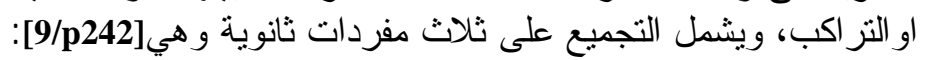




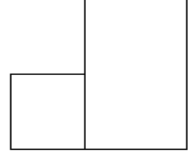

تلاصق

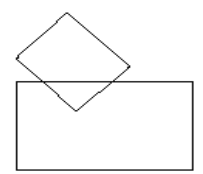

تر اكب

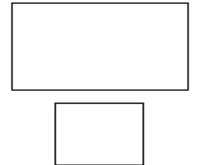

فصل

الثكل(6) : العلاقات التركيبية بين الأشكال [22/p412].

1. تلاصق الوحدات units adjoin: هو علاقة بين الوحدات بحيث تكون الوحدات ظاهرة ومرئية ومقرو عة ككيانات

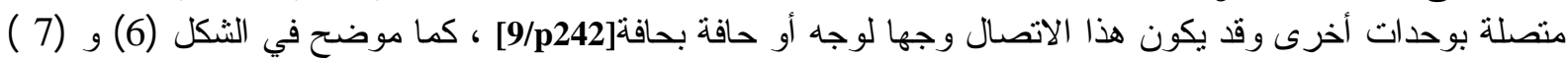
، فقد أوضح Baykan and Fox أن علاقة التلاصق هي من العلاقات التكوينية وهذه العلاقة تعني اشتر الك الوحدة بالحدود مع وحدة أخرى [5/p249].
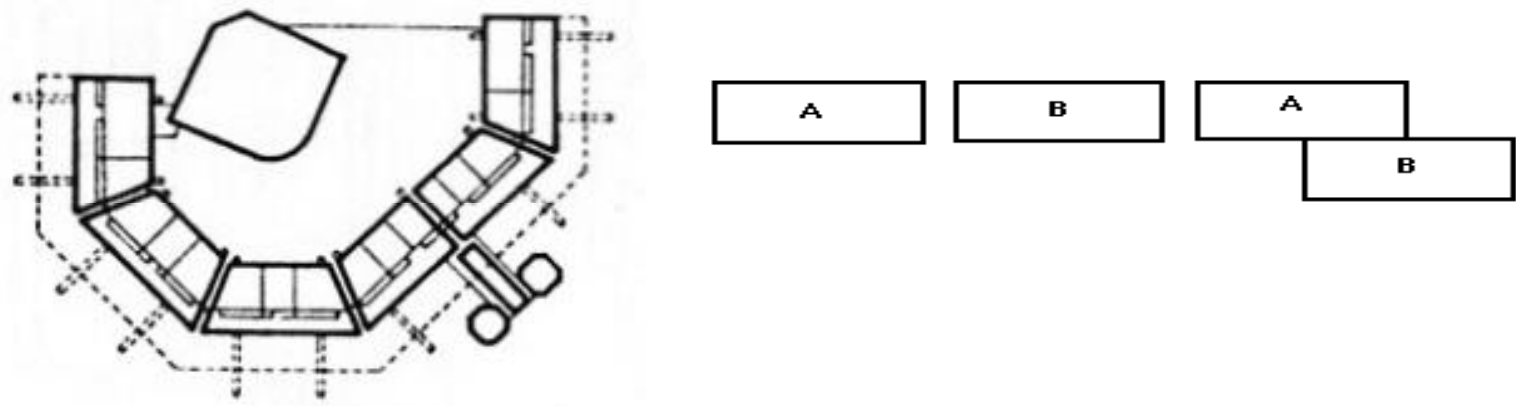

الشكل(7) مبنى 24/p242] FLorey Buliding].

الثكل(6): علاقة التلاصق[5/p249].

2.تراكب الوحدات units overlap: هو علاقة بين الوحدات في البعد ألحجمي بما يحقق التداخل

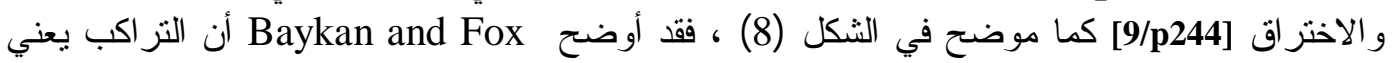
انثتر الك شكلين بمساحة معينة [5/24/p249]. 3. فصل الوحدات units separate: هو علاقة بين الوحدات المنفصلة عن بعضها ماديا أو معنويا

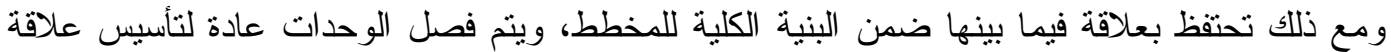

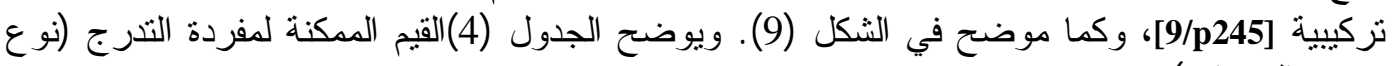

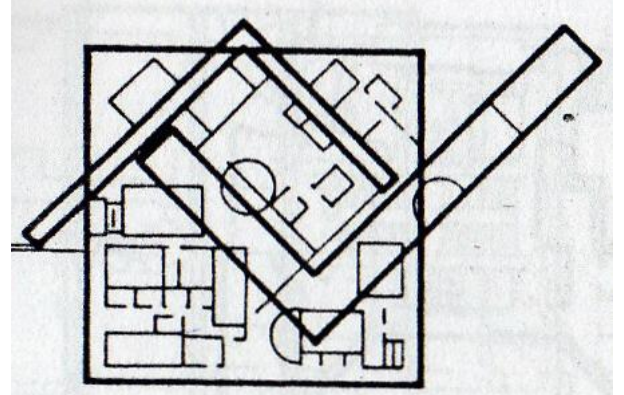

Occupational Health Center الثكل (8) مبنى

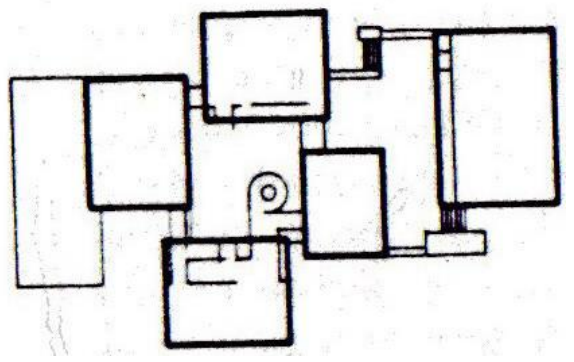

الثكل(9) مبنى [5/p249] Everson Museum Of Art. 
العاني : التكوين الشكلي للخانات في العمارة الإسلامية (دراسة تحليلية)

الجدول (4) : القيم الممكنة لمفردة التدرج (نوع تجميع الوحدات) [المصد:الباحث].

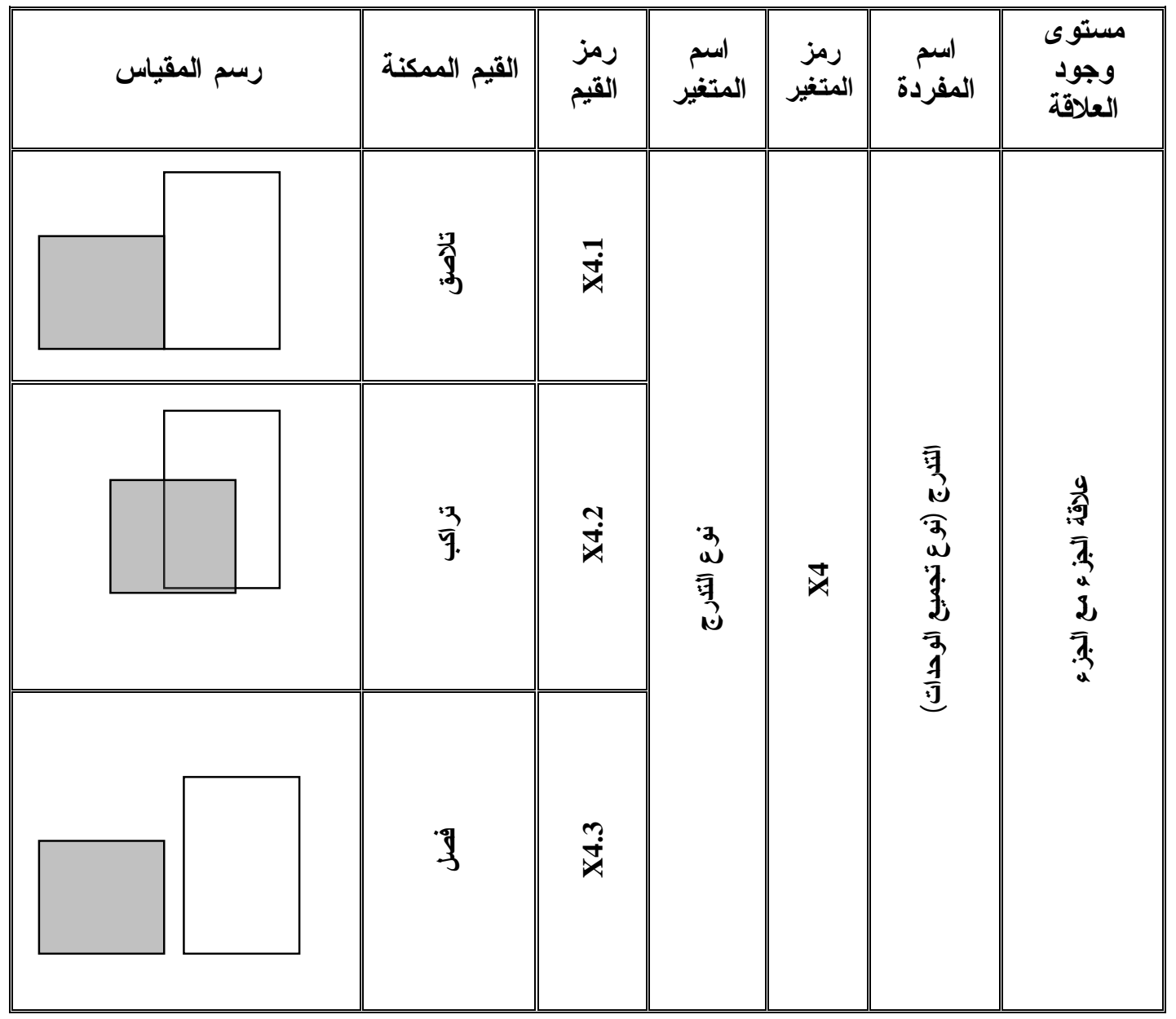

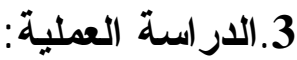

بعد أن تم تحديد المفردات الخاصة بالعلاقات التكوينية بين الوحدات سيتم تحيد الإجراءات العملية الممهدة

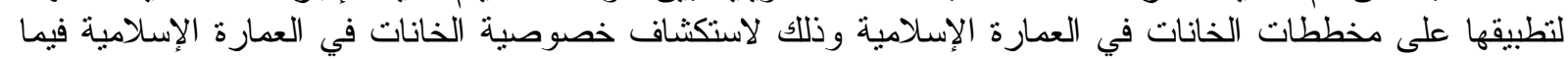
يخص تلاك المفردات.

1.3

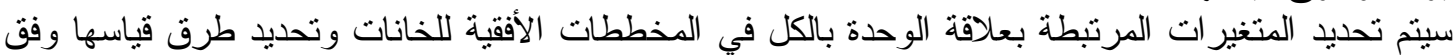

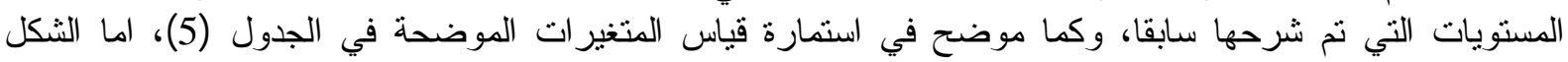

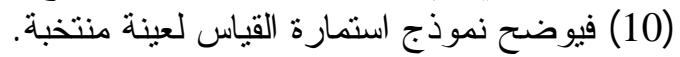

2.3 حدود الار اسة العملية:

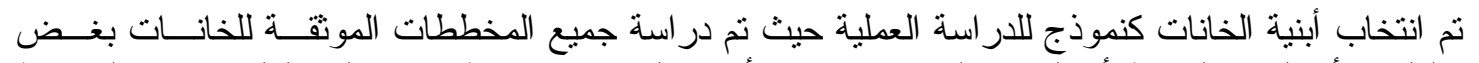

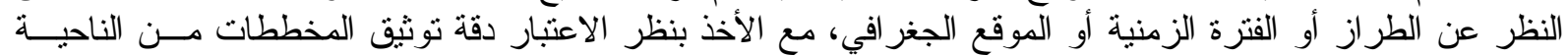

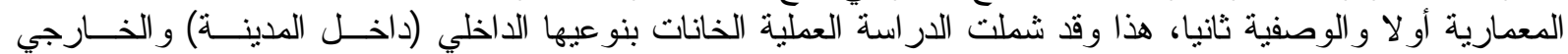
(الموجود على الطرق الخارجية) وكما نم شرحها في مقدمة البحث. 
الجدول (5) : تصميم استمارة القياس [الدصد:البحث].

\begin{tabular}{|c|c|c|c|}
\hline & & & اسم الخان \\
\hline & & & رقم الخان \\
\hline & & & موقع الخان \\
\hline القيم الممكنة & رمز القيم & اسم المتغير & رمز المتغير \\
\hline المخطط يشكل وحدة بحد ذاتها & $\overline{\mathbf{X} 1.1}$ & الوحدة الإجمالية & \\
\hline المخطط لا يشكل وحدة بحد ذاتها & $\mathrm{X} 1.2$ & (الوحدة تساوي الكل) & $\mathbf{A I}$ \\
\hline المخطط عبارة عن وحدة متضمنة للوحدات التي بداخلها & X2.1 & & \\
\hline المخطط لا يضم جميع الوحدات المكونة له & X2.2 & & \\
\hline الثكل النهائي للمخطط اكبر من مجموع الوحدات المكونة له & $\begin{array}{l}\mathbf{X 3 . 1} \\
\end{array}$ & & \\
\hline الثكل النهائي للمخطط مساوي لمجموع الوحدات المكونة له & $\mathrm{X3.2}$ & الأجزاء & \\
\hline تلاصق & X4.1 & & \\
\hline تراكب & $\mathbf{X} 4.2$ & التـرج & $\mathrm{X} 4$ \\
\hline فضصل & $\overline{\mathbf{X} 4.3}$ & & \\
\hline
\end{tabular}

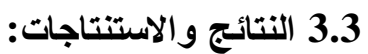

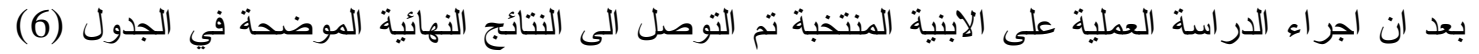
لجميع نماذج الخانات المنتخبة داخل نسيج المدينة، الما الجدول (7) فيوضح النتائج النهائية لجميع نماذج الخانية النانيات المنتخبة خارج نسيج المدينة. 
العاني : التكوين الشكلي للخانات في العمارة الإسلامية (در اسة تحليلية)

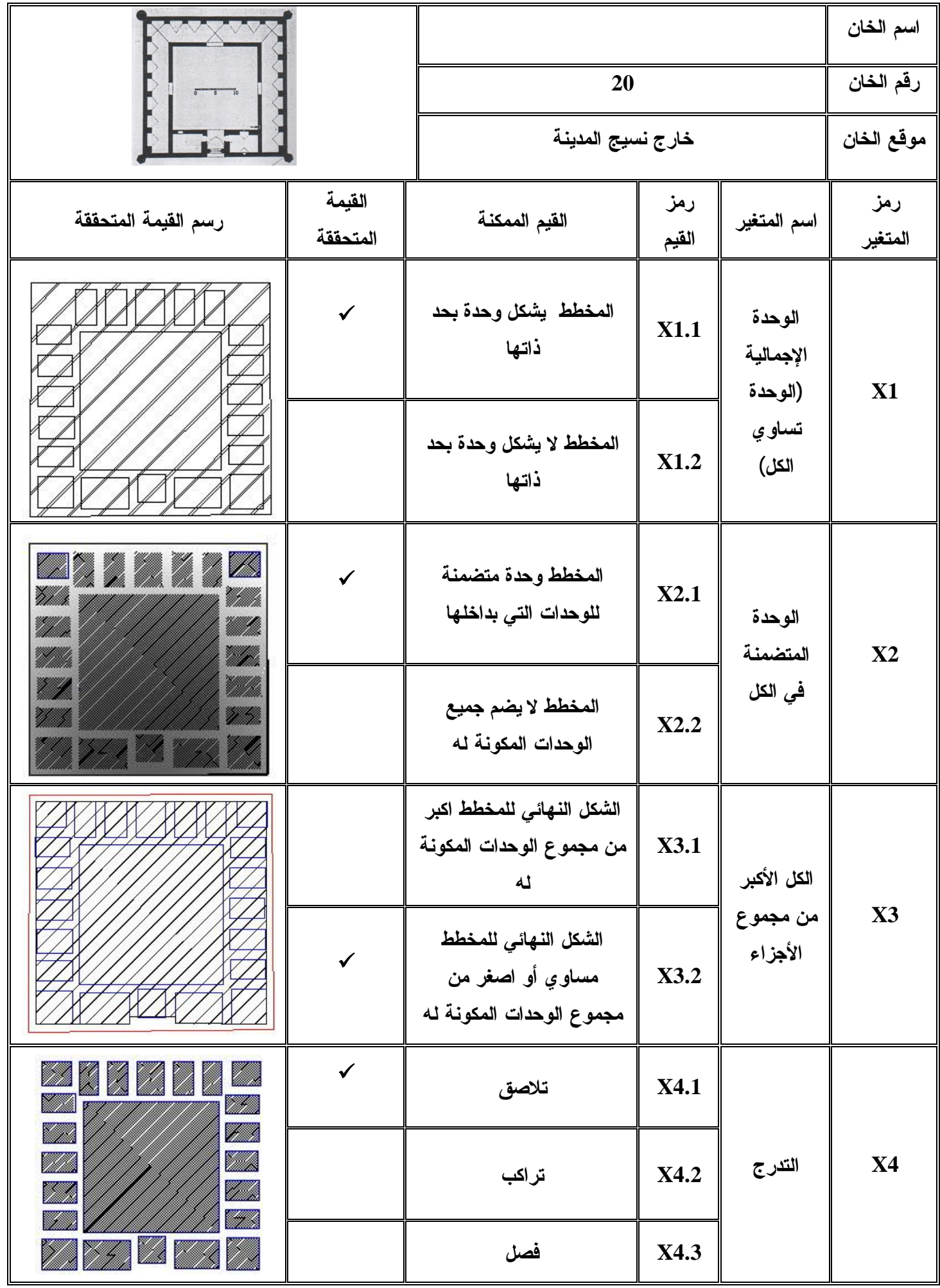

الثكل (10) نموذج استمارة القياس لعينة منتخبة [المصدر:الباحث]. 
$\begin{array}{llll}\text { Al-Rafidain Engineering } & \text { Vol.17 } & \text { No.4 } & \text { August } 2009\end{array}$

الجدول (6) النتائج النهائية لجميع نماذج الخانات المنتخبة (داخل نسيج المدينة) [المصد:الباحث].

\begin{tabular}{|c|c|c|c|c|c|c|c|c|c|}
\hline \multicolumn{3}{|c|}{ الت الترج } & \multicolumn{2}{|c|}{ الكل الأكبر من مجموع الأجزاء } & \multicolumn{2}{|c|}{ الوحدة متضمنة بكاملها } & \multicolumn{2}{|c|}{ (الوحدة تساؤي الإجماليةل } & \multirow{2}{*}{ الخان } \\
\hline $\mathbf{x 4 . 3}$ & $\begin{array}{c}\mathrm{x4.2} \\
\end{array}$ & $\begin{array}{l}\mathbf{x 4 . 1} \\
\end{array}$ & $\overline{\mathrm{X33.2}}$ & 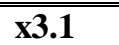 & $\begin{array}{c}\mathrm{X} 2.2 \\
\end{array}$ & $8 \mathrm{x2.1}$ & $\begin{array}{c}\mathrm{x} 1.2 \\
\end{array}$ & $\begin{array}{l}\mathrm{x1.1} \\
\end{array}$ & \\
\hline$\overline{\overline{0} \boldsymbol{0}}$ & $\overline{\overline{0} \boldsymbol{0}}$ & $\overline{11}$ & 1 & $\overline{\overline{0}}$ & $\overline{\overline{0}}$ & $\overline{11}$ & $\overline{\overline{0}}$ & $\overline{11}$ & c1 \\
\hline 0 & 0 & 1 & 1 & 0 & 0 & 1 & 0 & 1 & c5 \\
\hline 0 & 0 & 1 & 1 & 0 & 0 & 1 & 0 & 1 & c7 \\
\hline 0 & 0 & 1 & 1 & 0 & 0 & 1 & 0 & 1 & $\begin{array}{l}\mathbf{c 1 0} \\
\end{array}$ \\
\hline 0 & 0 & 1 & 1 & 0 & 0 & 1 & 0 & 1 & c11 \\
\hline 0 & 0 & 1 & 1 & 0 & 0 & 1 & 0 & 1 & c12 \\
\hline 0 & 0 & 1 & 1 & 0 & 1 & 0 & 0 & 1 & c13 \\
\hline 0 & 0 & 1 & 1 & 0 & 0 & 1 & 0 & 1 & c14 \\
\hline 0 & 0 & 1 & 1 & 0 & 0 & 1 & 0 & 1 & c15 \\
\hline 0 & 1 & 1 & 0 & 1 & 0 & 1 & 1 & 0 & c24 \\
\hline 0 & 1 & 1 & 1 & 0 & 0 & 1 & 0 & 1 & c25 \\
\hline 0 & 0 & 1 & 0 & 1 & 0 & 1 & 1 & 0 & c26 \\
\hline 0 & 0 & 1 & 1 & 0 & 0 & 1 & 0 & 1 & c27 \\
\hline 0 & 1 & 1 & 1 & 0 & 0 & 1 & 1 & 0 & c28 \\
\hline 0 & 0 & 1 & 1 & 0 & 0 & 1 & 0 & 1 & c29 \\
\hline 1 & 0 & 1 & 0 & 1 & 0 & 1 & 0 & 1 & c30 \\
\hline 0 & 0 & 1 & 1 & 0 & 0 & 1 & 0 & 1 & c31 \\
\hline 0 & 0 & 1 & 0 & 1 & 1 & 0 & 0 & 1 & c32 \\
\hline 0 & 0 & 1 & 1 & 0 & 1 & 0 & 0 & 1 & $\mathbf{c 3 3}$ \\
\hline 0 & 0 & 1 & 1 & 0 & 0 & 1 & 0 & 1 & c34 \\
\hline 1 & 0 & 1 & 0 & 1 & 1 & 0 & 0 & 1 & c35 \\
\hline 0 & 0 & 1 & 1 & 0 & 0 & 1 & 0 & 1 & $\mathrm{c36}$ \\
\hline 0 & 1 & 1 & 1 & 0 & 1 & 0 & 1 & 0 & c37 \\
\hline 0 & 0 & 1 & 1 & 0 & 0 & 1 & 0 & 1 & $\mathbf{c 3 8}$ \\
\hline 0 & 0 & 1 & 1 & 0 & 0 & 1 & 0 & 1 & $\begin{array}{l}\text { c39 } \\
\end{array}$ \\
\hline 0 & 0 & 1 & 1 & 0 & 0 & 1 & 0 & 1 & c40 \\
\hline 0 & 0 & 1 & 1 & 0 & 0 & 1 & 0 & 1 & c41 \\
\hline 0 & 0 & 1 & 1 & 0 & 0 & 1 & 0 & 1 & c42 \\
\hline 0 & 0 & 1 & 1 & 0 & 0 & 1 & 0 & 1 & c43 \\
\hline 1 & 0 & 1 & 1 & 0 & 1 & 0 & 1 & 0 & c44 \\
\hline 0 & 0 & 1 & 1 & 0 & 1 & 0 & 1 & 0 & c45 \\
\hline 0 & 0 & 1 & 1 & 0 & 0 & 1 & 0 & 1 & c46 \\
\hline 0 & 1 & 1 & 1 & 0 & 1 & 0 & 1 & 0 & c47 \\
\hline 0 & 0 & 1 & 1 & 0 & 0 & 1 & 0 & 1 & c48 \\
\hline 0 & 0 & 1 & 1 & 0 & 0 & 1 & 0 & 1 & $\begin{array}{l}\mathrm{cto} \\
\mathrm{c} 49\end{array}$ \\
\hline 0 & 0 & 1 & 1 & 0 & 0 & 1 & 0 & 1 & c50 \\
\hline $8 \%$ & $14 \%$ & $100 \%$ & $86 \%$ & $14 \%$ & $22 \%$ & $78 \%$ & $19 \%$ & $81 \%$ & التحبة" \\
\hline
\end{tabular}

الجدول (7) النتائج النهائية لجميع نماذج الخانات المنتخبة (خارج نسيج المدينة) [ المصدر:الباحث].

\begin{tabular}{|c|c|c|c|c|c|c|c|c|c|}
\hline \multicolumn{3}{|c|}{ الترج } & \multicolumn{2}{|c|}{ الكل الأكبر من مجموع الأجزاء } & \multicolumn{2}{|c|}{ الوحدة متضمنة بكاملها } & \multicolumn{2}{|c|}{ (الوحدة الإجمالية } & \multirow{2}{*}{ الخان } \\
\hline $\mathrm{x} 4.3$ & $\mathrm{x} 4.2$ & $\mathrm{x} 4.1$ & x3.2 & x3.1 & $\mathrm{x} 2.2$ & $\mathrm{x} 2.1$ & $\mathbf{x 1 . 2}$ & x1.1 & \\
\hline 0 & 0 & 1 & 1 & 0 & 0 & 1 & 0 & 1 & c2 \\
\hline $\mathbf{0}$ & $\mathbf{0}$ & 1 & 1 & $\mathbf{0}$ & 0 & 1 & 0 & 1 & c3 \\
\hline $\mathbf{0}$ & $\mathbf{0}$ & 1 & 1 & 0 & 0 & 1 & $\mathbf{0}$ & 1 & c4 \\
\hline $\mathbf{0}$ & $\mathbf{0}$ & 1 & 1 & $\mathbf{0}$ & $\mathbf{0}$ & 1 & $\mathbf{0}$ & 1 & c6 \\
\hline $\mathbf{0}$ & $\mathbf{0}$ & 1 & 1 & $\mathbf{0}$ & $\mathbf{0}$ & 1 & $\mathbf{0}$ & 1 & $\mathrm{c8}$ \\
\hline $\mathbf{0}$ & $\mathbf{0}$ & 1 & 1 & $\mathbf{0}$ & $\mathbf{0}$ & 1 & 0 & 1 & c9 \\
\hline $\mathbf{0}$ & 0 & 1 & 1 & $\mathbf{0}$ & $\mathbf{0}$ & 1 & 0 & 1 & c16 \\
\hline $\mathbf{0}$ & $\mathbf{0}$ & 1 & 1 & $\mathbf{0}$ & $\mathbf{0}$ & 1 & 0 & 1 & c17 \\
\hline $\mathbf{0}$ & $\mathbf{0}$ & 1 & 1 & $\mathbf{0}$ & $\mathbf{0}$ & 1 & $\mathbf{0}$ & 1 & c18 \\
\hline $\mathbf{0}$ & $\mathbf{0}$ & 1 & 1 & $\mathbf{0}$ & $\mathbf{0}$ & 1 & $\mathbf{0}$ & 1 & c19 \\
\hline $\mathbf{0}$ & $\mathbf{0}$ & 1 & 1 & $\mathbf{0}$ & $\mathbf{0}$ & 1 & 0 & 1 & c20 \\
\hline $\mathbf{0}$ & $\mathbf{0}$ & 1 & 1 & $\mathbf{0}$ & $\mathbf{0}$ & 1 & $\mathbf{0}$ & 1 & c21 \\
\hline 0 & 0 & 1 & 1 & 0 & 0 & 1 & 0 & 1 & $\mathbf{c 2 2}$ \\
\hline 0 & 0 & 1 & 1 & 0 & 0 & 1 & 0 & 1 & c23 \\
\hline $0 \%$ & $0 \%$ & $100 \%$ & $100 \%$ & $0 \%$ & $0 \%$ & $100 \%$ & $\mathbf{0 \%}$ & $100 \%$ & التحقبّة \\
\hline
\end{tabular}


1.3.3مناقشة نتائج التحليل الثكلي للنماذج المعمارية (المخططات):

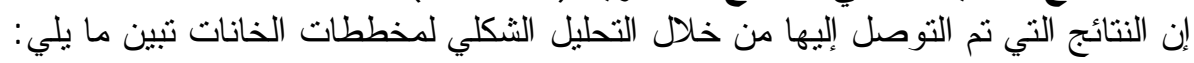

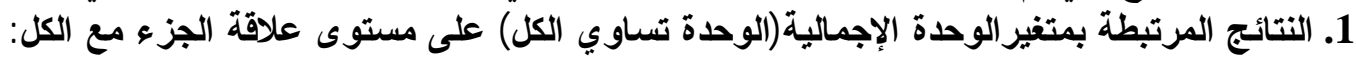

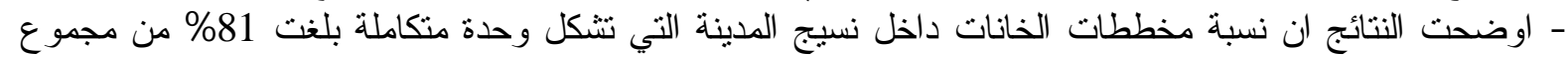

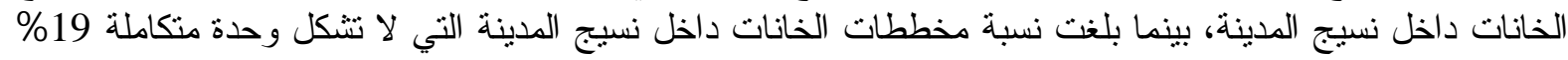
فقط من المجمو ع داخل نسيج المدينة. - اوضحت النتائج ان نسبة مخططات الخانات خار جن نسيج المدينة التي نشكل وحدة منكاملة بلغت 100\% من مجموع الخانات خار ج نسيج المدينة و الثنكل (11) يوضنح هذه انت النتائج.
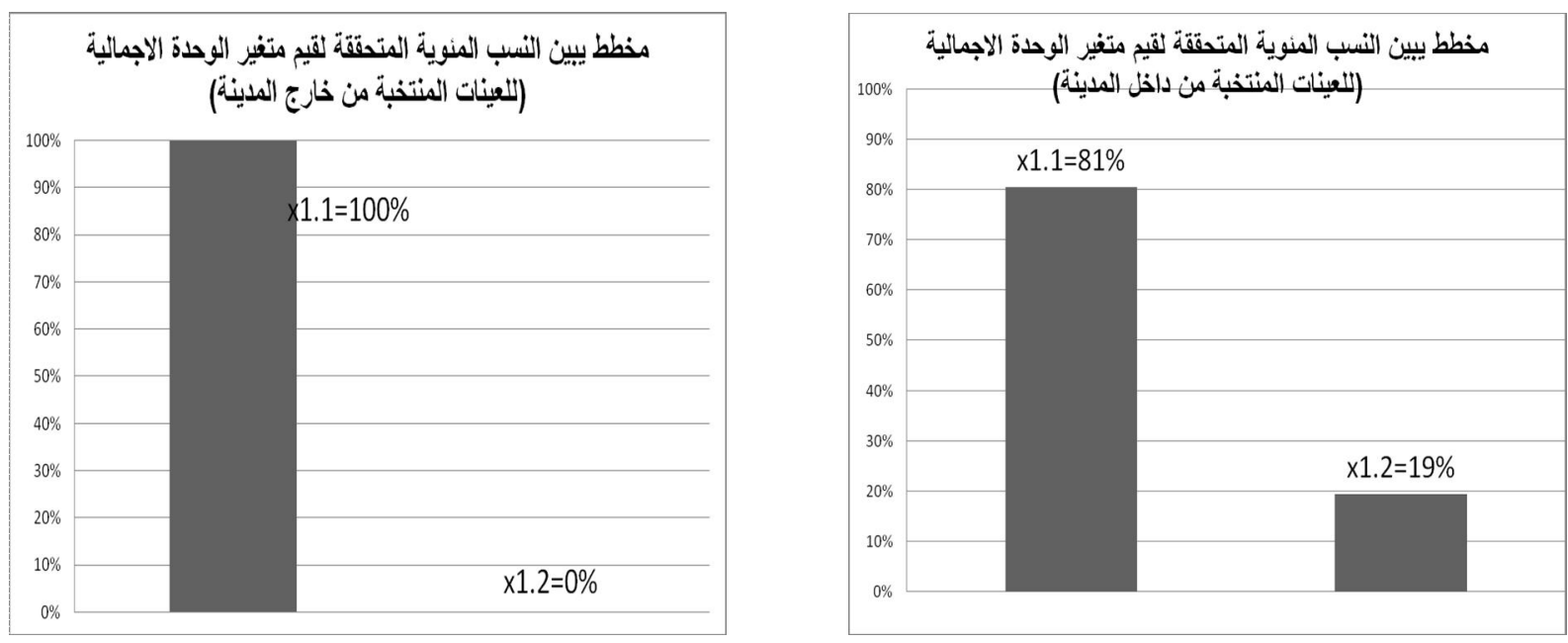

الشكل (11) :النتائج المرتبطة بمتغير الوحدة الإجمالية(الوحدة نساوي الكل) [المصدر:الباحث].

2. النتائج المرتبطة بمتغير الوحدات المتضمنة في الكل على مستوى علاقة الجزء مع الكل:

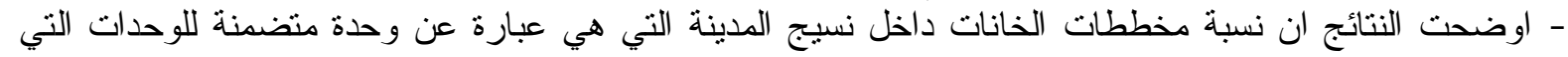

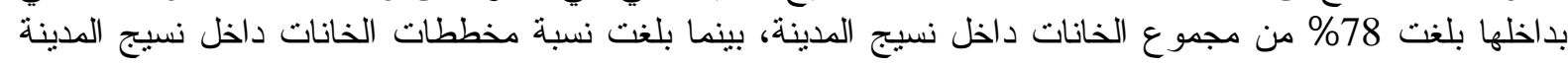

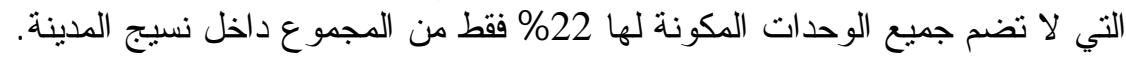

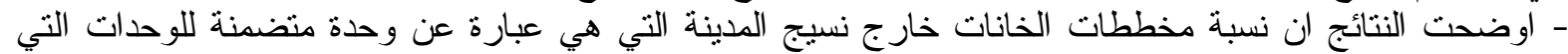

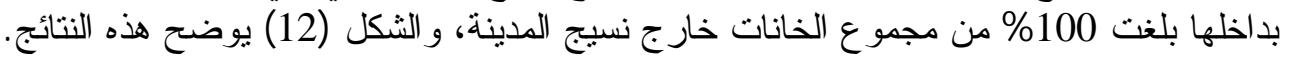
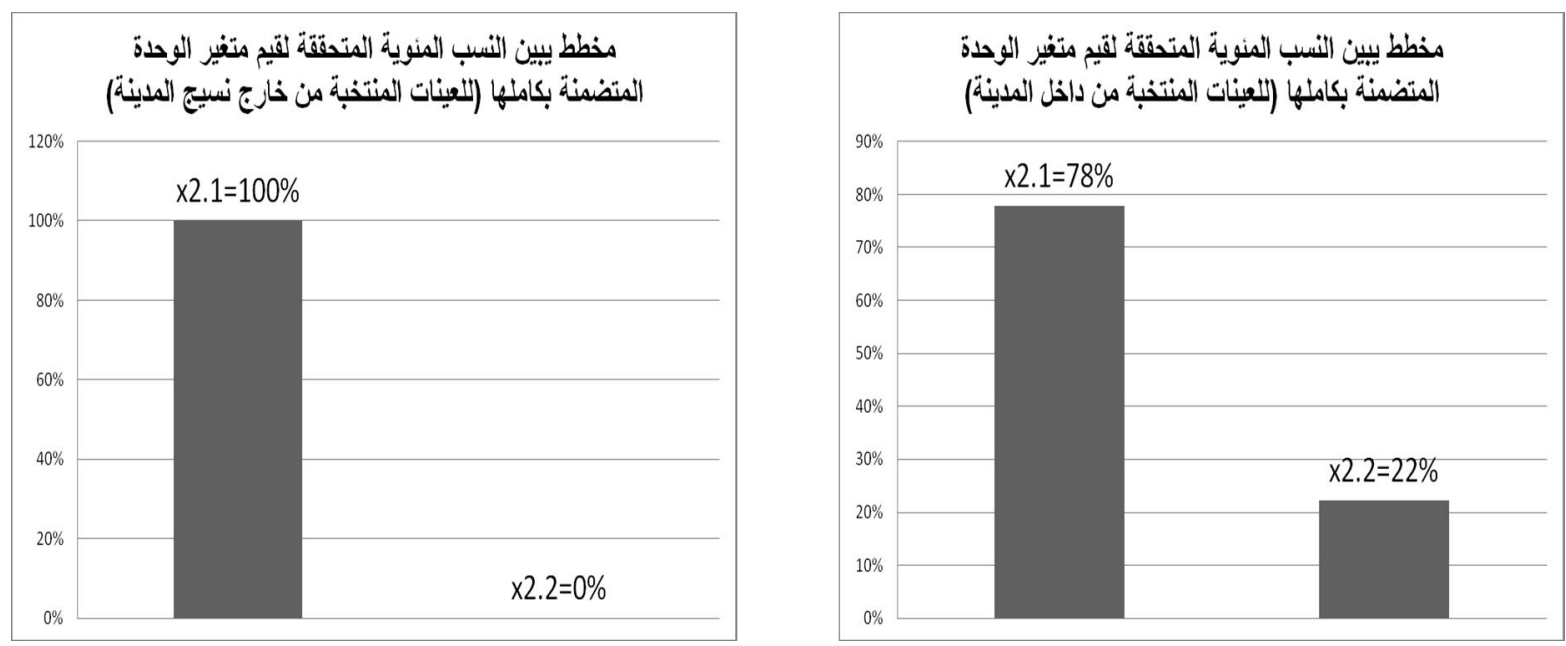

الشكل (12):النتائج المرتبطة بمتغير الوحدات المتضمنة في الكل[المصدر:الباحث]. 
$\begin{array}{llll}\text { Al-Rafidain Engineering } & \text { Vol.17 } & \text { No.4 } & \text { August } 2009\end{array}$

3. النتائج المرتبطة بمتفير الكل الأكبر من مجموع الأجزاء على مستوى علاقة الجزء مع الكل:

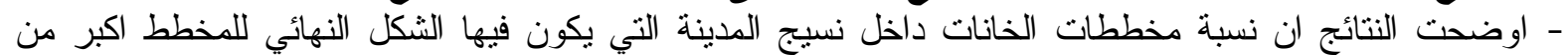

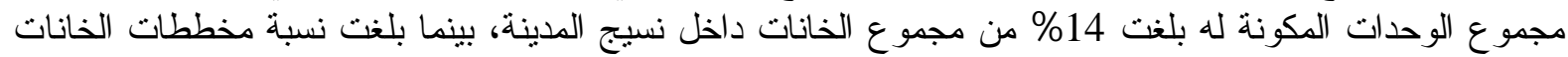
داخل نسيج المدينة الني يكون فيها الثكل النهائي للمخطط مساوي لمجموع الوحدات المكونة له 86\% من المجموع داخل نسيج المدينة. - اوضحت النتائج ان نسبة مخططات الخانات خارج نسيج المدينة التي يكون فيها الثكل النهائي للمخطط مساوي

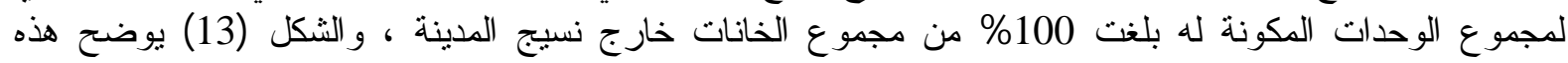
النتائج.

4. النتائج المرتبطة بمتغير التدرج على مستوى علاقة الجزءء مع الجزءء:

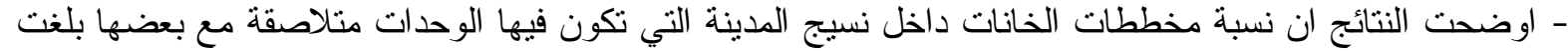
100\% من مجموع الخانات داخل نسيج المدينة، بينما بلغت نسبة مخططات الخانات داخل نسيج المدينة التي تكون

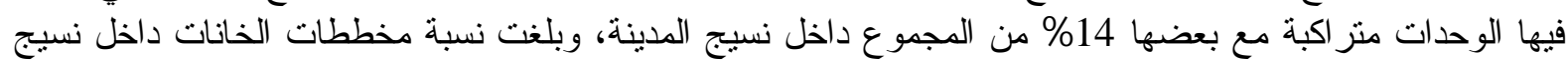

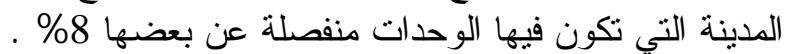

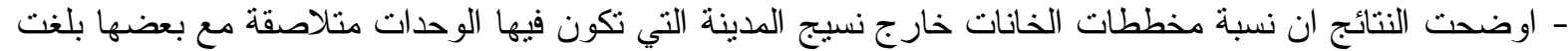
100\% من مجموع الخانات خارج نسيج المدينة، بينما كانت نسب المخططات خارج نسيج المدينة التي نكون فيها الوحدات متز اكبة او منفصلة عن بعضها 00\% و و الثكل (14) يوضح هذه النتائج.
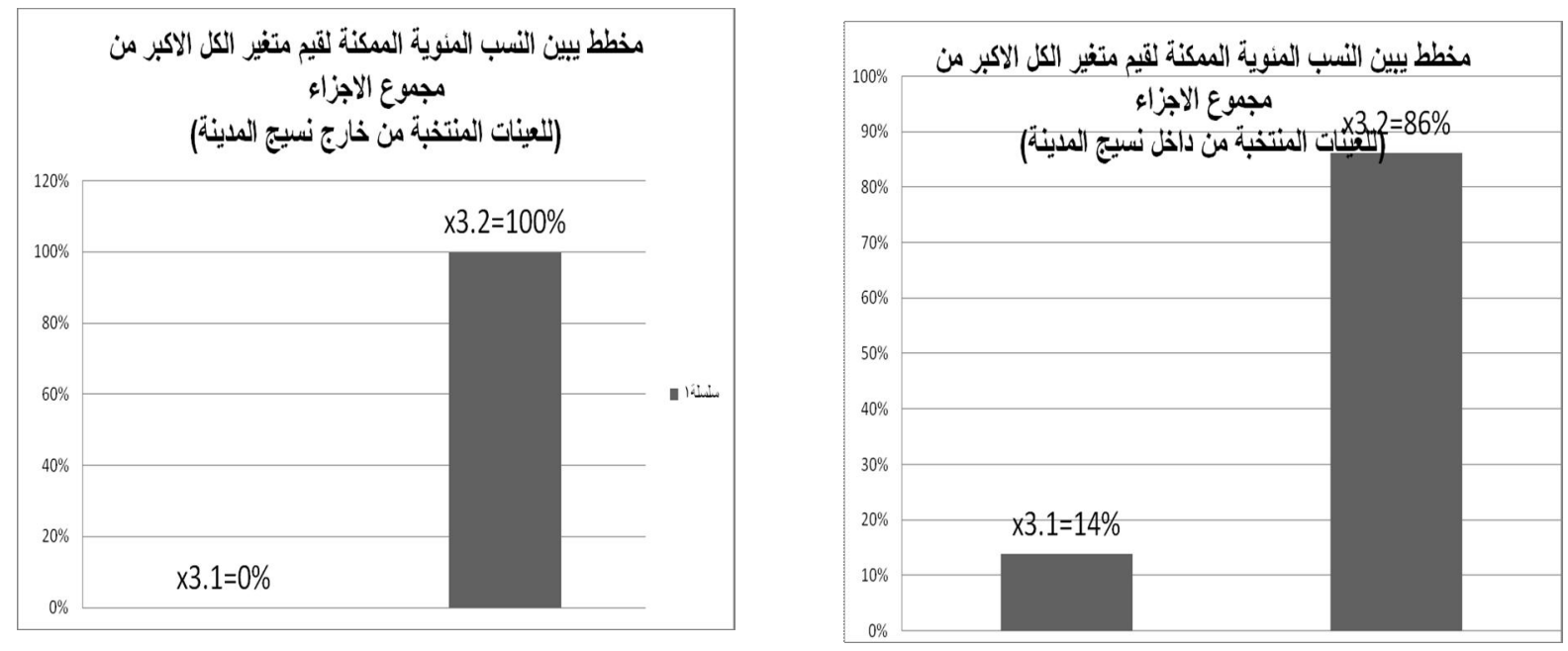

الثكل (13):النتائج المرتبطة بمتغير الكل الأكبر من مجموع الأجز اء[(لمصدر:الباحث].

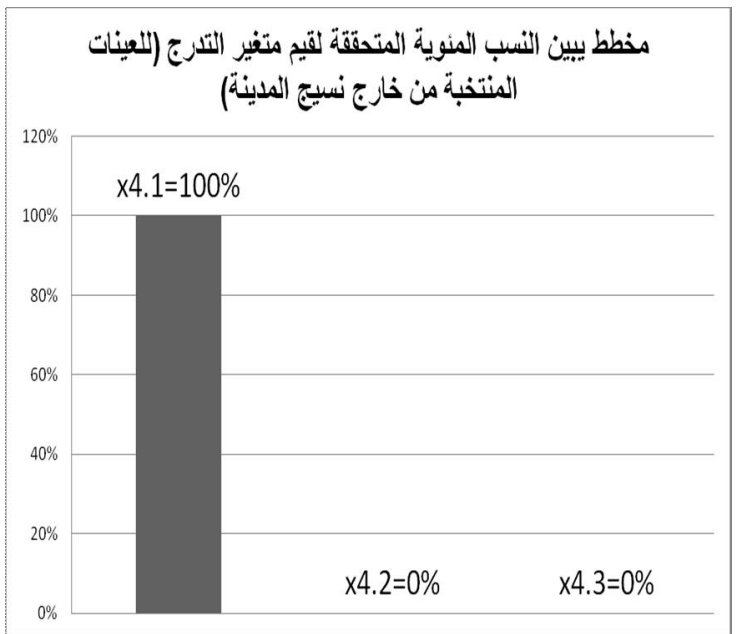

مخطط ييين النسب الموية المتحقةة لقيم متفير التلدج (للعينات المنتفبة من داخل نسيج المدينة)

الثكل (14):النتائج المرتبطة بمتغير التدر ج[المصدر:الباحث].

2.3.3 تحليل نتائج التحليل الثكلي للنماذج المعمارية (المخططات) إحصائيا: 
يتضمن تطبيق عملية تحليل نتائج التحليل الثنكلي للنماذج(المخططات) المعمارية إحصائيا استخدام تحليل قوة

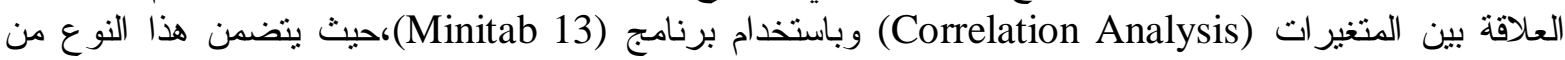

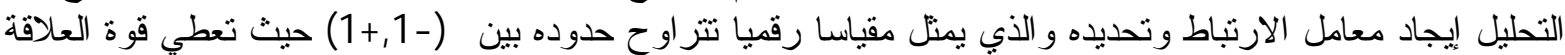

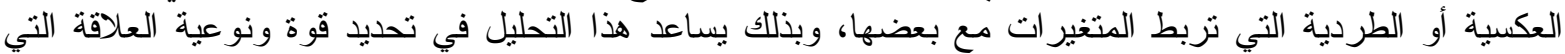

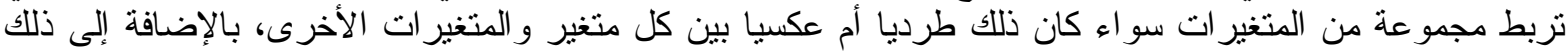

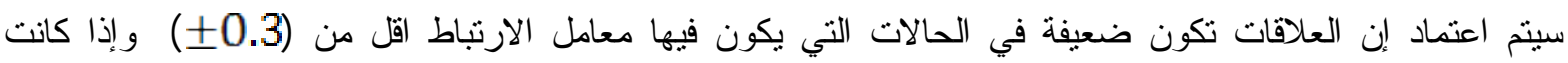

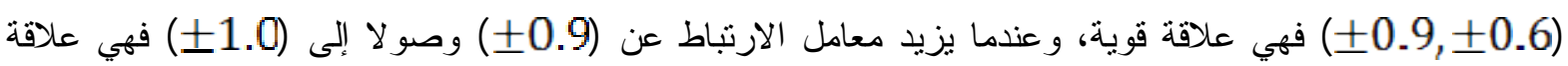
قوية جدا.

إن النتائج التي نم التوصل إليها من خلال التحليل الإحصائي و المنتنل بقياس معامل الارتباط وقوة العلاقة

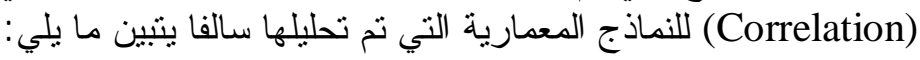

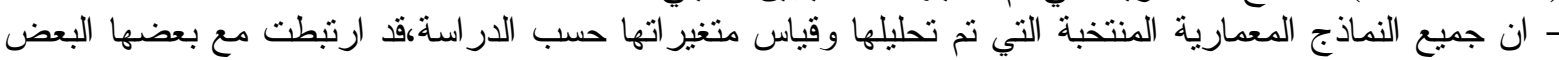

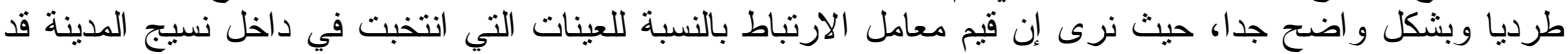

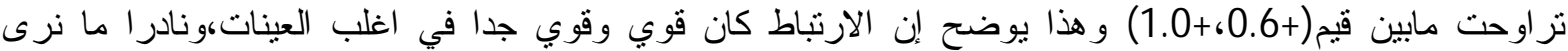
ارنباطات عكسية أو متوسطة أو ضعيفة.

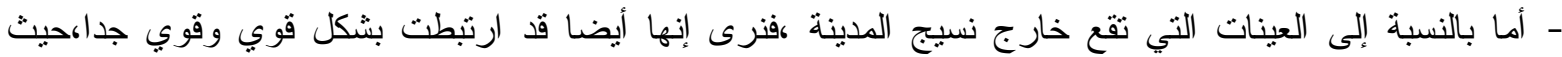

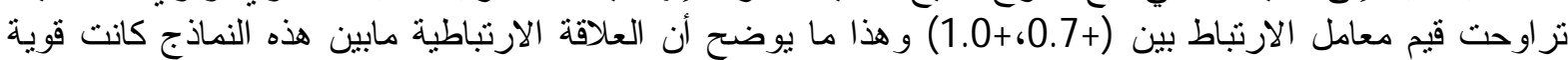
جدا كولم نلاحظ علاقة عكسية أو ضعيفة.

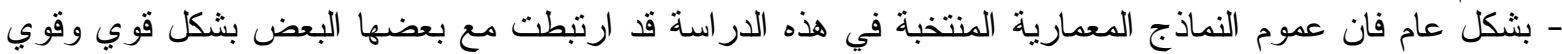

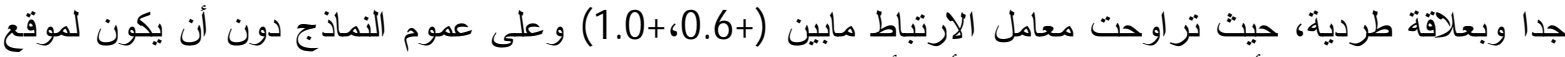

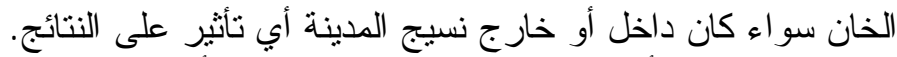

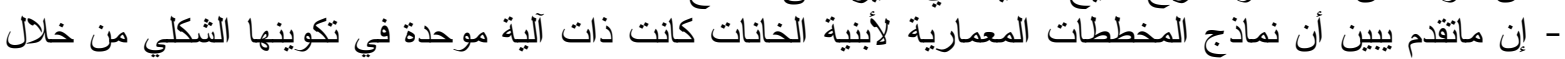

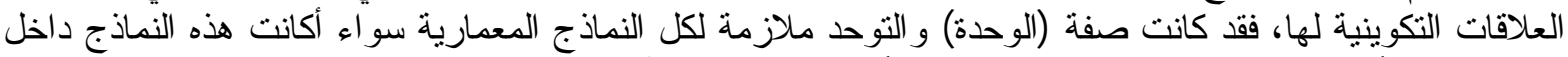

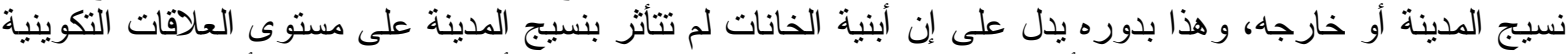
للمخطط، وهذا بدوره يشير إلى إن أبنية الخانات في العمارة الإسلامية كانت أبنية (نمطية)، إي أن هذه النية المخططات

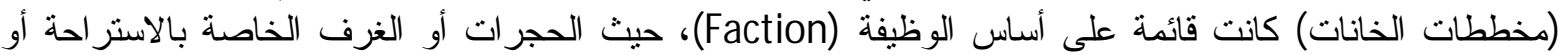

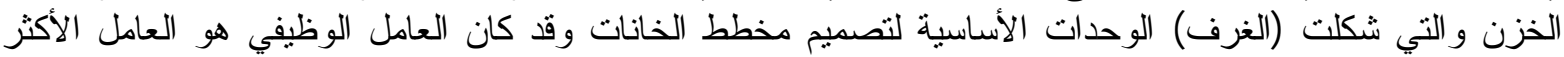
تأثير ا على تشكيل هذا النمط من الأبنية الإسلامية.

3.3.3 الاستنتاجات النهائية:

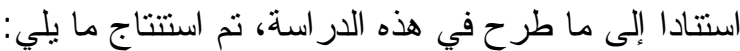

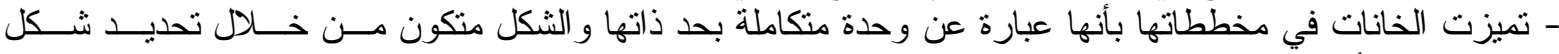

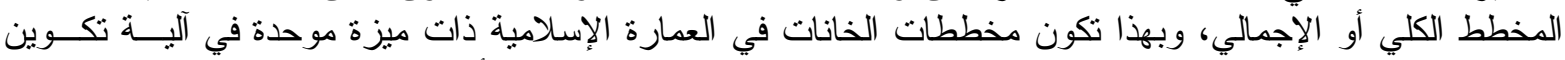

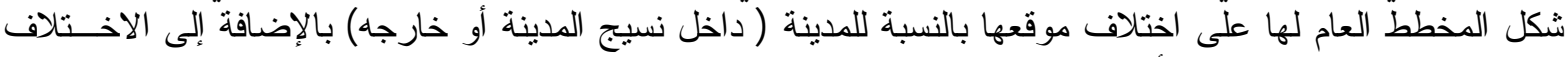

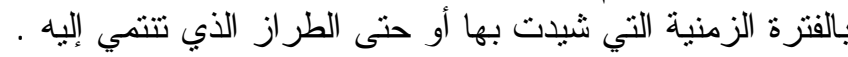

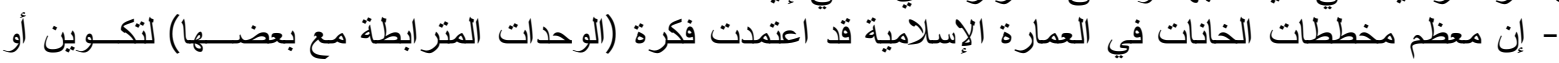
تشكيل قياس البناية إجمالا.

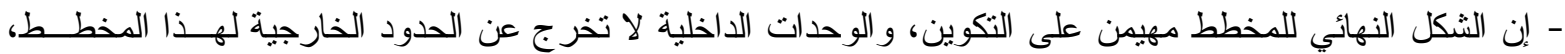

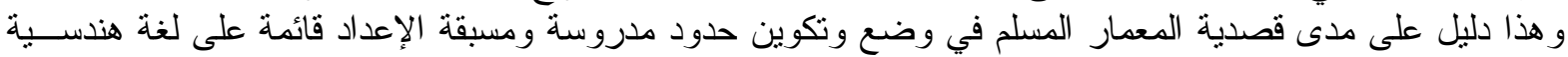

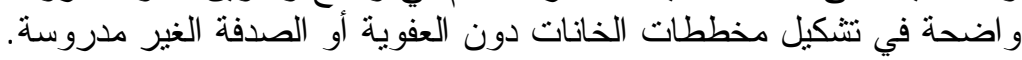

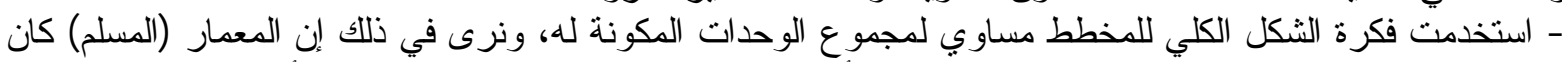

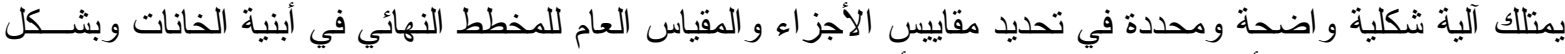

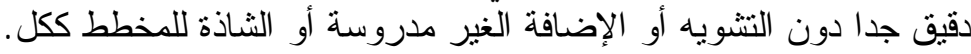

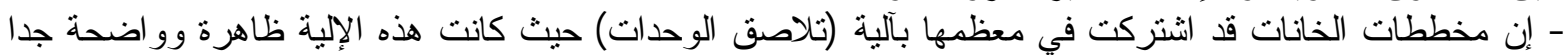

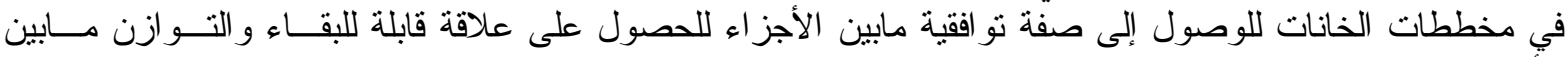
الأجز اء في المخطط الكلي للخان كنو ع وظيفي نمطي في العمارة الإسلامية . لإنين 
$\begin{array}{lllll}\text { Al-Rafidain Engineering } & \text { Vol.17 } & \text { No.4 } & \text { August } 2009\end{array}$

- ـ أن التكوين الشكلي لمخططات أبنية الخانات في العمارة الاسلامية قد استتدت على مجموعة من العلاقات التكوينيــة

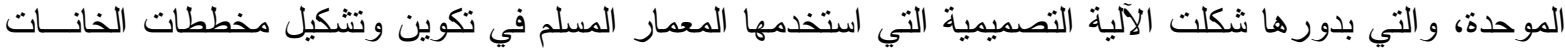

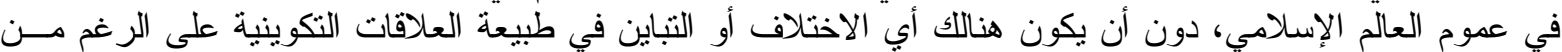

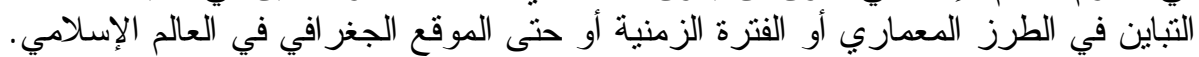

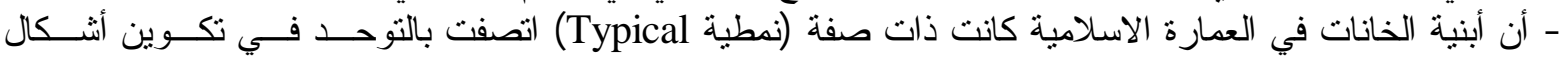
مخططاتها (Plans)،سواء أكانت هذه المخططات داخل نسيج المدينة أو خارجها ،ويأتي ذللك من تأثنير العامل الوظيفي

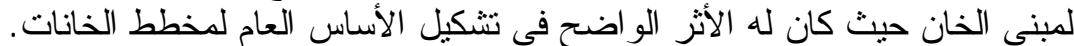

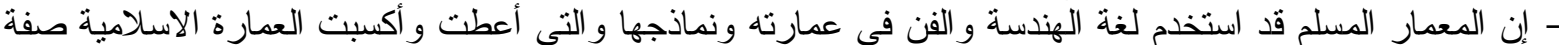

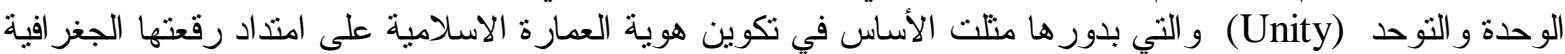

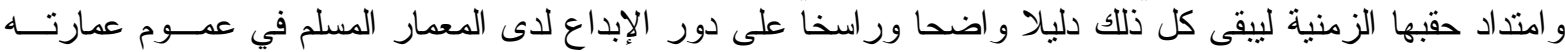
بدافع وحدة فكره الحضاري الذي تمتع بـا أين ما كان وحيثما كان.

1 المصادر : بهنسي، عفيف، "العمارة العربية ، الجمالية ، الوحدة ، والتنوع" المجلس القومي للنقافة العربية، الرباط ، المغرب، .1990 2 - العمري، حفصة رمزي،" اثر الدين الاسلامي على تثكيل انماط ابنية العمران، مع دراسة تحليلية لنمط المساجد

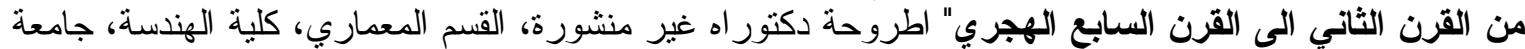
بغداد، 2000 .

3 - المالكي، قبيلة فارس،" التناسب و المنظومات التناسبية في العمارة العربية الاسلامية - جراسة تعليلية للعبات العمارة

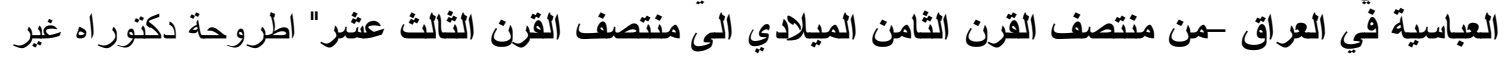

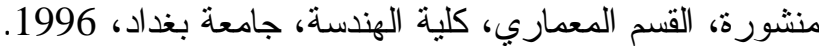

4- Ardalan, Nader, \& Bakhtiar Laleh, "The Sense Of Unity -The Sufi Tradition In Prsian Architecture", The University of Chicago Press, Chicago \& London, (1973)

5- Baykan, C. A. and Fox, M. S., "Spatial synthesis by disjunctive constraint satisfaction", Artificial Intelligence in Engineering Design, 11, (1997).

6- Bonta, Juan, "Notes On The Theory Of Meaning In The Design", John \& Willy, New York, (1980).

7- Chase S. C., "Modeling Designs With Shape Algebras and Formal Logic", Ph.D dissertation, University of California, Los Angeles(1996).

8- Ching, Francis D.K, “Arch. Form, Space and Order”, Van Nstrrand Reinhold Company, (1996).

9-Clark R.H. and Pause M. , “ Precedents in Architecture. ,Analytic Diagrams Formative Ideas and Partis"John Wiley \& Sons. ,United States of America(2005).

10- Emdanant Samir S., "An Ontology for Conceptual Design in Architecture", Ph.D. in Architecture College of Architecture and Urban Planning ,The University of Michigan Ann Arbor, Michigan, 48109-2069, USA, (1998).

11 -Gero, J.S. and Jo, J.H., "Space Layout Planning Using an Evolutionary Approach", Artificial Intelligence in Engineering, 12(3), (1995).

12- Gero, J. S., "Shape pattern recognition using a computable shape pattern representation", Artificial Intelligence in Design '98, Kluwer, Dordrecht, (1998).

13- Grube, Ernest, "What Is Islamic Architecture", Architecture Of Islamic World, George Michell Ceditor,Thames \& Hudson, London, (1991).

14- Hillenbrand, Robret, "Islamic Architecture, Form, Function and Meaning" „Edinburgh University Press, (1994).

15- Hillier, Bill, "Space Is The Machine", Cambridge University Press Cambridge, (1996).

16- Holod,Renata, "Text, Plan \& Building- On The Transmission Of Architectural Knowwledge- Theories And Principles Of Design In The Architecture Of Islamic 
Societies",A Symposium held by the aga Khan program for Islamic Architecture, Harvard Unv. \& Massachusetts Institute of Technology, Cambridge, Massachusetts, (1984)

17- Medjdoub B., Richens P.and Barnard N., "Building Services Standard Solutions", Computer Aided Design, 40, (2001).

18- Michalek, J., "Interactive layout design optimization", MS.C. Thesis, University of Michigan, (2001).

19- Rosenman M.A., "The Generation of Form Using Evolutionary Approach", AI in Design'96, J.S. Gero and F. Sudweeks (eds), Kluwer Academic, Netherlands, (1996).

20- Steadman, J. P., "Architecture Morphology ", Pion Ltd., London, (1983).

21- Stiny,G., "An Evaluation of Palladian Plans", Environment and Planning B, Vol.5, (1978).

22-Stiny G., "Kindergarten grammars: designing with Froebel's building gifts" Environment and Planning B: Planning and Design, Vol. 7, (1980b) .

23- Tabbaa, Yasser, " Geometry \& Memory In The Design Of The Madrasat Al-Firdows

In Aleppo- Theories And Principles Of Design In The Architecture Of Islamic Societies", A Symposium held by the aga Khan program for Islamic Architecture, Harvard Unv. \& Massachusetts Institute of Technology, Cambridge, Massachusetts, (1988).

24-Tansey, Richard G, " Art Through The Age", Harcourt Brace Jovavich, College

Publishers, (1991).

25-Thompson, D. Arcy," On Growth \& Form" , Cambridge University -Massachusetts, U.S.A, (1971).

26-Zevi,Bruno, " Architecture As A Space-How To Look At Architecture" ,DA CAPO

Press, New York, (1993).

تم اجراء البحث في كلية الهندسة - جامعة الموصل 